\title{
Decision strategies in the ABX (matching-to-sample) psychophysical task
}

\author{
MICHAEL J. HAUTUS and XIANDONG MENG \\ University of Auckland, Auckland, New Zealand
}

\begin{abstract}
The ABX psychophysical task is very useful when the dimensions along which the stimulus varies cannot be readily described. However, there are a few problems associated with its use. First, the task has often been used with slight procedural variations, which hinders the extraction of a bias-free index of performance. Second, the most common index of sensitivity derived from this task is the ubiquitous proportion correct. Third, some researchers undervalue the importance that the decision strategy adopted by the observer has on estimates of sensitivity. We describe a standard methodology for the ABX task and outline models of performance based on two decision strategies. These models can be used to extract the detection-theoretic index of performance, $d^{\prime}$, from ABX data. The results of three $\mathrm{ABX}$ discrimination experiments, in which circle size, number value, and tone amplitude are used, are reported. Analysis of the decision spaces derived from observer responses indicates that these observers adopted a suboptimal difference decision strategy, even after they had received considerable practice with feedback. This suggests that, given no prior knowledge of the nature of the decision strategy adopted by an observer, the difference model of ABX discrimination could be a good choice when observer performance is estimated.
\end{abstract}

The $\mathrm{ABX}^{1}$ psychophysical task shares with only a handful of other standard tasks, such as the same-different and oddity tasks, the characteristic that it is ideal for the testing of naive observers, or experienced observers when the stimuli are multidimensional. The reason this group of tasks is so useful is that the observer need have no knowledge about the physical dimensions along which the stimuli vary. Indeed, in many instances, even the experimenter does not possess this information.

The literature abounds with accounts of the use of the ABX task across fields within psychology as diverse as psycholinguistics (e.g., Dupoux, Pallier, Sebastian, \& Mehler, 1997) and animal research (e.g., Spence, 1937). Unfortunately, the exact implementation of the task varies across studies. Furthermore, few researchers have endeavoured to extract bias-free measures of performance from their particular implementation of the ABX task. The most commonly reported index of performance is the percentage of correct responses, $p(\mathrm{C})$ (e.g., Calder, Young, Perrett, Etcoff, \& Rowland, 1996; Etcoff \& Magee, 1992; Liberman, Harris, Hoffman, \& Griffith, 1957; Pastore, Li, \& Layer, 1990). This index is sensitive not only to the observer's discriminative ability, but also to the observer's response bias and the instructions given by the experimenter (Elliott, 1964; Tanner \& Birdsall, 1958). Another point against $p(\mathrm{C})$ is that no empirical receiver-

The authors thank John Irwin, David Noreen, and two anonymous reviewers for their valuable comments on earlier drafts of this manuscript. Correspondence concerning this article should be addressed to M. J. Hautus, Department of Psychology, University of Auckland, Private Bag 92019, Auckland, New Zealand (e-mail: m.hautus@auckland. ac.nz). operating characteristics (ROCs) have been found that conform to the implied ROC for this index (Swets, 1986).

In addition to identifying and using a bias-free index of performance, it is also critically important to determine the decision strategy that is used by an observer making judgments in an ABX task. There are two main types of strategy that can be adopted: a difference strategy (Pierce \& Gilbert, 1958) or an independent-observations strategy (Macmillan, Kaplan, \& Creelman, 1977). To employ a model based on a strategy that was not used by the observer will result in incorrect estimates of sensitivity. The difference between the estimates of sensitivity obtained by assuming one model or the other is not trivial (see Macmillan \& Creelman, 1991, Table A5.3).

We first outline a standard methodology for the ABX task. This methodology is developed as an extension of the structure of other psychophysical tasks, such as the yes/no ${ }^{2}$ and forced-choice tasks. Noreen (1981) and Macmillan and Creelman (1991, Chap. 7) described the ABX task in a manner similar to that given here.

In the $\mathrm{ABX}$ task, there are two events to be discriminated, $S_{1}$ and $S_{2}$, that may vary along any number of physical dimensions. Three stimulus presentations are made within a trial at each of the three observation locations (either spatial or temporal), A, B, and X. Thus, A, B, and $X$ are not specific events. One stimulus arising from $S_{1}$ and another from $S_{2}$ are presented randomly at locations $\mathrm{A}$ and $\mathrm{B}$. These stimuli are often referred to as the standards. Either $S_{1}$ or $S_{2}$ is presented at location $\mathrm{X}$, with an equal probability of presentation for each. This stimulus at $\mathrm{X}$ is called the focus. The task for the observer is to match the focus to the location that contains a standard stimulus that arose from the same event as the focus. This may 
be accomplished by a simple binary decision (e.g., the match is to $\mathrm{A}$ or to $\mathrm{B}$ ) or by a confidence rating (e.g., rating confidence on a 6-point scale that the focus was a match to A). Given this structure for the $\mathrm{ABX}$ task, there are four possible stimulus sequences: $\left\langle\mathrm{S}_{1}, \mathrm{~S}_{2}, \mathrm{~S}_{1}\right\rangle$ and $<\mathrm{S}_{2}, \mathrm{~S}_{1}, \mathrm{~S}_{2}>$, for which the correct response is "A," and $<\mathrm{S}_{1}, \mathrm{~S}_{2}, \mathrm{~S}_{2}>$ and $<\mathrm{S}_{2}, \mathrm{~S}_{1}, \mathrm{~S}_{1}>$, for which the correct response is " $\mathrm{B}$."

\section{The Difference Decision Strategy}

For the difference decision strategy, the approach adopted by the observer is to calculate the differences between the evidence arising from $\mathrm{X}$ and the evidence arising from $\mathrm{A}$ and $\mathrm{B}$. A decision is then made on the basis of the two differences, using a likelihood-ratio criterion. An unbiased observer would adopt a likelihoodratio criterion of $\beta=1$, which corresponds to selecting the location that yields the evidence that is the most similar to that in $\mathrm{X}$ - that is, the location that produced the smallest difference. A biased observer would adopt a criterion other than $\beta=1$. For a criterion of $\beta=2$, for example, an observer would only select $A$ if, on the basis of the evidence, it were at least twice as likely to be the match to $\mathrm{X}$ as was B. Unlike the case for an unbiased observer, there is no simple interpretation of the strategy adopted by a biased observer.

A simple example may be used to illustrate this decision strategy. Suppose that an unbiased judge is required to discriminate between children who are in Room $8\left(\mathrm{~S}_{1}\right)$ and those who are in Room $10\left(\mathrm{~S}_{2}\right)$ at a local primary school. The only information available to the judge is the height of the children. Furthermore, suppose that the average heights of the children in Rooms 8 and 10 are 110 and $120 \mathrm{~cm}$, respectively. Now, the heights of three children are presented to the judge: 100,125 , and $114 \mathrm{~cm}$. The judge is told that, of the first two children, one is from Room 8 and the other from Room 10. The judge's task is to determine whether the third child (the focus) is from the same room as the first child or the second child. First, the judge, using a difference decision strategy, determines the absolute difference in height between the first and the third children $(|100-114|=14 \mathrm{~cm})$ and the second and the third children $(|125-114|=11 \mathrm{~cm})$. As the absolute difference in height between the first and the third children $(14 \mathrm{~cm})$ is larger than that between the second and the third children $(11 \mathrm{~cm})$, the judge will determine that the third child came from the same room as the second child.

Notice how the judge does not use all of the information available-in particular, the mean heights of the children in each room. Would the use of this information lead to better performance than ignoring it? The answer is yes. However, to do this requires another decision strategy, the independent-observations strategy, which yields optimal performance on the ABX task (Macmillan et al., 1977; Noreen, 1981). Before giving details of this "optimal" strategy, we will consider further the mathematical representation of the difference decision strategy.
The decision space for the difference decision strategy consists of the joint distributions of the differences between $\mathrm{X}$ and the other two locations. With four possible event sequences, there are four possible difference distributions. Under the assumption of normally distributed sensory evidence with a variance of one, the difference distributions will have a variance of two. For each event sequence, there are two relevant differences to be calculated, and hence, the difference distributions are bivariate with a variance of two. Each of the two differences in each sequence is based on $\mathrm{X}$, and hence, the bivariate distributions of differences are correlated with covariance, unity.

Abramowitz and Stegun (1972, p. 936) have provided a useful representation of the fully parameterized bivariatenormal distribution. Substitution of the above parameters into their Equation 26.3.12 yields a general equation for the difference distributions:

$$
\begin{aligned}
f_{\mu_{x_{1}}, \mu_{x_{2}}} & = \\
& \frac{1}{\sqrt{12} \pi} e^{-\frac{1}{3}\left(\left(x_{1}-\mu_{x_{1}}\right)^{2}-\left(X_{1}-\mu_{x_{1}}\right)\left(x_{2}-\mu_{x_{2}}\right)+\left(X_{2}-\mu_{x_{2}}\right)^{2}\right)},
\end{aligned}
$$

where $X_{1}$ is the difference between the evidence arising from $\mathrm{A}$ and $\mathrm{X}$ and $X_{2}$ is the difference between the evidence arising from $\mathrm{B}$ and $\mathrm{X}$. The means, $\mu_{x_{1}}$ and $\mu_{x_{2}}$, of the two difference distribution can take three possible values: zero, $d^{\prime}$, and $-d^{\prime}$. The mean sensory effects produced by the two events, $\mathrm{S}_{1}$ and $\mathrm{S}_{2}$, are equal to zero and $d^{\prime}$, respectively.

A hit in the ABX task can be defined as selecting $A$ as the match when it is the match, whereas a false alarm can be defined as selecting $\mathrm{A}$ as the match when it is not. From these definitions and Equation 1, the likelihoodratio function can be calculated ${ }^{3}$ :

$$
\begin{aligned}
\beta\left(X_{1}, X_{2}, d^{\prime}\right) & =\frac{f_{0, d^{\prime}}+f_{0,-d^{\prime}}}{f_{-d^{\prime}, 0}+f_{d^{\prime}, 0}} \\
& =\frac{e^{\frac{1}{3} d^{\prime}\left(X_{1}-X_{2}\right)}\left(e^{\frac{4}{3} d^{\prime} X_{2}}+e^{\frac{2}{3} d^{\prime} X_{1}}\right)}{e^{\frac{4}{3} d^{\prime} X_{1}}+e^{\frac{2}{3} d^{\prime} X_{2}}} .
\end{aligned}
$$

Although Equation 2 is reasonably compact, it becomes rather unwieldy when rearranged to obtain either $X_{1}\left(X_{2}, d^{\prime}\right.$, $\beta$ ) or $X_{2}\left(X_{1}, d^{\prime}, \beta\right)$. These two functions can be obtained in closed form but are not reported here because of their size.

Given Equations 1 and 2, the decision space for the ABX task under the assumption of a difference decision strategy can be depicted. Figure 1, analogous to that of Macmillan and Creelman (1991, Figure 7.2, p. 168), illustrates this decision space. The ellipses represent contours of equal probability density for each bivariate distribution; their shape is due to the covariance between the two dimensions. The thicker lines illustrate various criterial boundaries. Three separate pairs of boundaries are 


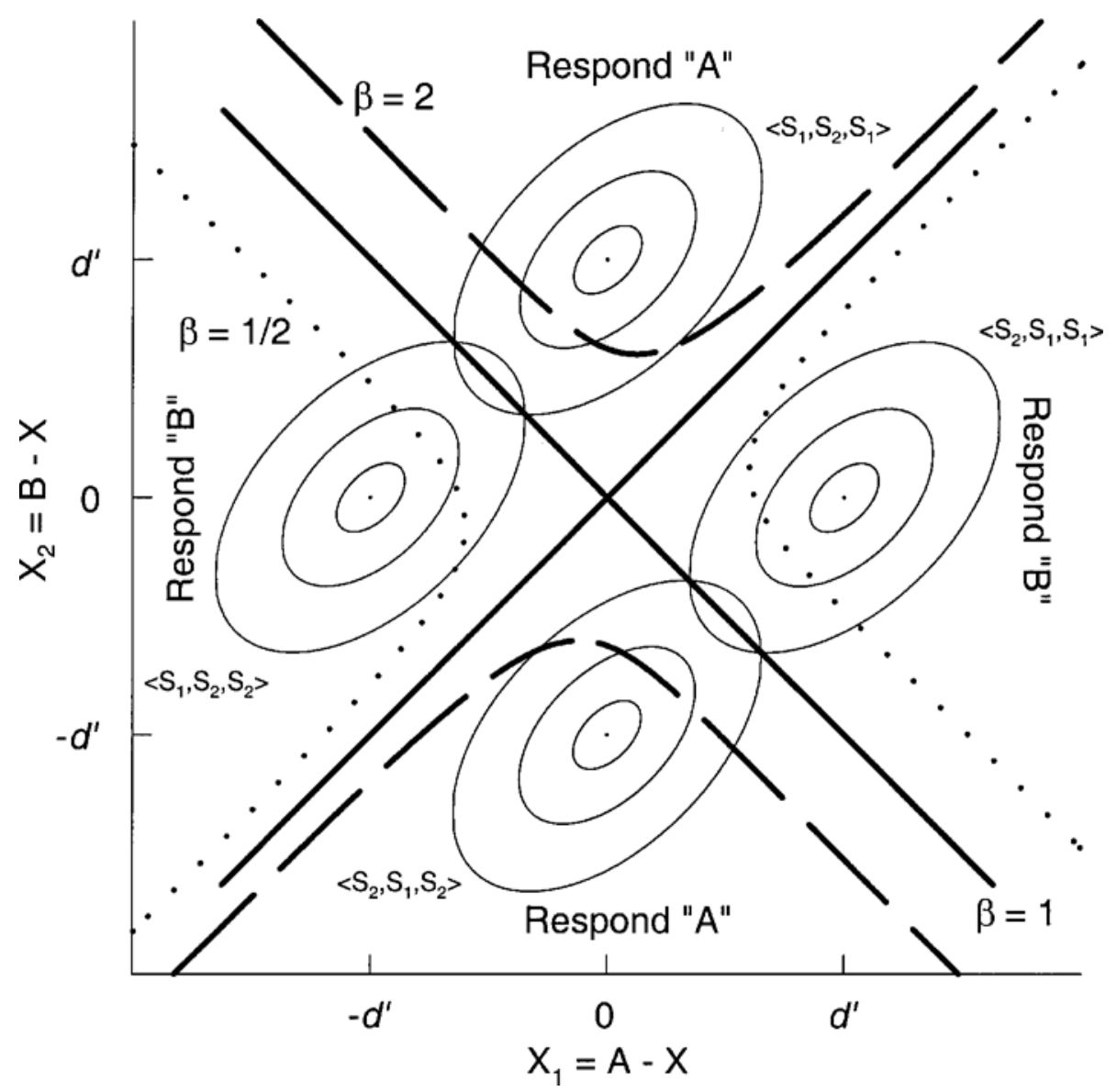

Figure 1. The decision space for the difference strategy in the ABX task. The axes represent the differences between the evidence arising from observations $A$ and $B$ and the focus, $X$. The ellipses illustrate contours of equal likelihood for the distributions of evidence arising from the four stimulus sequences. The heavy lines are criterial boundaries that divide the decision space into different response regions. The depicted boundaries correspond to likelihood ratios of 0.5 (dotted), 1.0 (solid), and 2 (dashed).

illustrated. They correspond to three different values of $\beta(0.5,1.0$, and 2.0$)$ and a fixed value of $d^{\prime}(2.0)$. These particular values of $\beta$ were selected for two purposes. The first was to illustrate the boundaries adopted by an unbiased observer $(\beta=1)$. For this case, the boundaries are straight lines with slopes of plus-and-minus one. The second purpose was to illustrate the symmetry that arises between the boundaries formed by a particular likelihood ratio (e.g., $\beta=2$ ) and the boundaries formed by the reciprocal of that likelihood ratio (e.g., $\beta=1 / 2$ ). This example demonstrates how the criterial boundaries for $\beta=x$ are the same as those for $\beta=1 / x$ reflected about the line $X_{2}=X_{1}$.

For an unbiased observer, the decision space is segregated into four regions. In Figure 1, there are two regions labeled "Respond A" and two regions labeled "Respond B." These labels represent the responses that an observer would give on the basis of the region within which the evidence on a trial falls. For an observer who adopts a likelihood ratio greater than unity, there are two "Respond B" regions and one "Respond A" region. The two "Respond A" regions for an unbiased observer become merged into one larger region when $\beta>1$. The situation is reversed for an observer who adopts a likelihood ratio less than one.

The false alarm rate, $F$, is equal to the proportion of the total volume under the difference distributions $f_{-d^{\prime}, 0}$ and $f_{d^{\prime}, 0}$ that falls within the regions labeled "Respond A" in Figure 1. Two equations for the false alarm rate are required: one for $\beta \geq 1$, the other for $\beta \leq 1$ :

$$
\begin{aligned}
& \text { if } \beta \geq 1, \quad F=\int_{-\infty}^{\infty} \int_{X_{2}\left(\beta, X_{1}, d^{\prime}\right)}^{\infty} f_{-d^{\prime}, 0}+f_{d^{\prime}, 0} d X_{2} d X_{1} \\
& \text { if } \beta \leq 1, \quad F=\int_{-\infty}^{\infty} \int_{X_{1}\left(\beta, X_{2}, d^{\prime}\right)}^{\infty} f_{-d^{\prime}, 0}+f_{d^{\prime}, 0} d X_{1} d X_{2} .
\end{aligned}
$$

The hit rate, $H$, is equal to the proportion of the total volume under the difference distributions $f_{0,-d^{\prime}}$ and $f_{0, d^{\prime}}$ that falls above or below the criterial boundaries. Once again, two equations are required: 


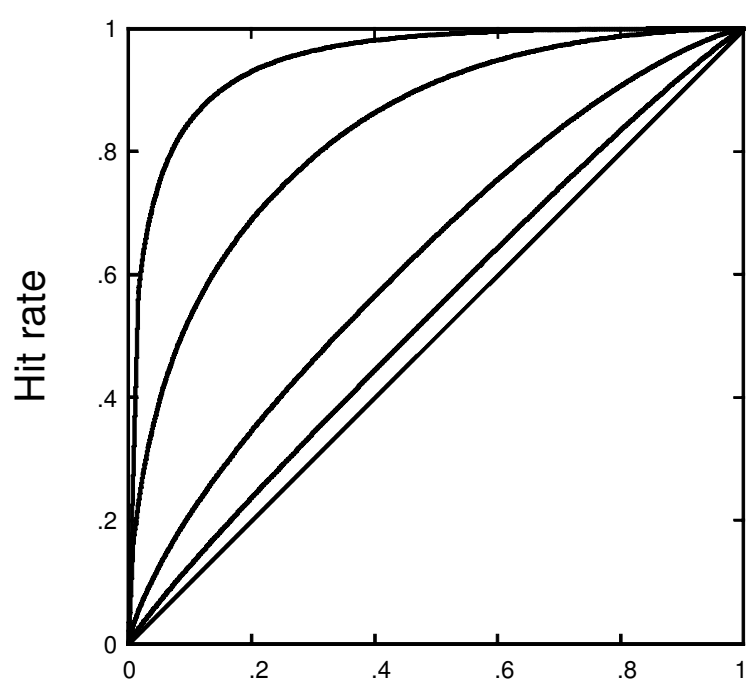

False-alarm rate

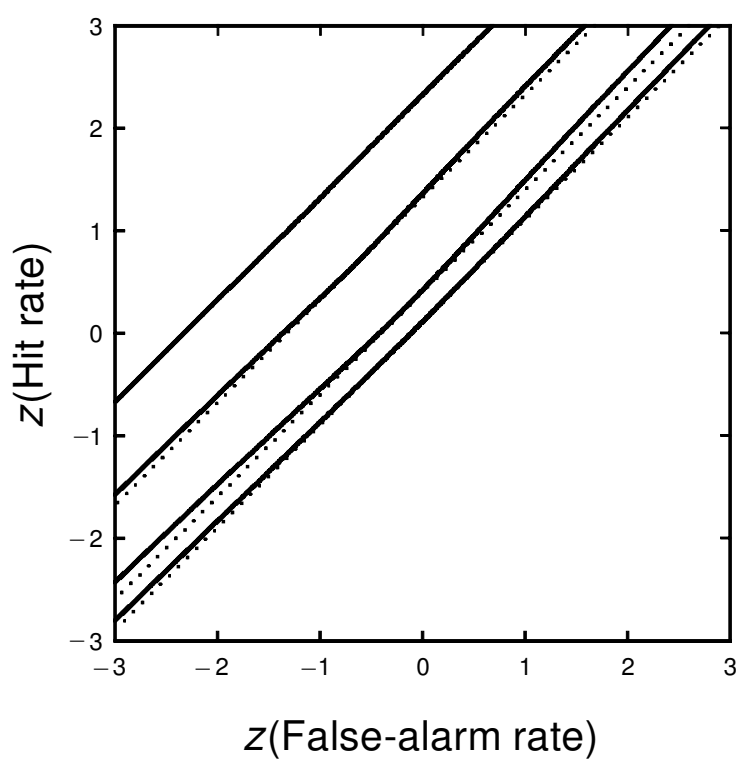

Figure 2. Theoretical receiver-operating characteristics (ROCs) for the difference strategy in the ABX task. The ROCs are illustrated in the unit square (left panel) and on inverse-normal coordinates (right panel). Values of $d^{\prime}$ are $0.5,1.0,2.0$, and 3.0. Lines of unit slope (dotted) have been added to the right panel to highlight the slight deviation of the ROCs from linearity.

$$
\begin{aligned}
& \text { if } \beta \geq 1, \quad H=\int_{-\infty}^{\infty} \int_{X_{2}\left(\beta, X_{1}, d^{\prime}\right)}^{\infty} f_{0,-d^{\prime}}+f_{0, d^{\prime}} d X_{2} d X_{1} \\
& \text { if } \beta \leq 1, \quad H=\int_{-\infty}^{\infty} \int_{X_{1}\left(\beta, X_{2}, d^{\prime}\right)}^{\infty} f_{0,-d^{\prime}}+f_{0, d^{\prime}} d X_{1} d X_{2} .
\end{aligned}
$$

Close examination of the decision space in Figure 1 reveals some interesting symmetries. As was mentioned above, the criterial boundaries for $\beta=1 / x$ are simply those for $\beta=x$ reflected about the line $X_{2}=X_{1}$. In addition, the proportion of the volume under the distributions $f_{0, d^{\prime}}$ and $f_{0,-d^{\prime}}$ that falls above and below the criterial boundaries for $\beta=x$ is equal to the proportion of the volume under the distributions $f_{-d^{\prime}, 0}$ and $f_{d^{\prime}, 0}$ that falls beyond (to the left and right) the criterial boundaries for $\beta=$ $1 / x$. This symmetry is proof that the ROC for the ABX task is symmetrical about the negative diagonal of the ROC space. Furthermore, it suggests the redundancy of one of the equations for $F$ and one of the equations for $H$, because it can be shown that

$$
H\left(d^{\prime}, \beta\right)=1-F\left(d^{\prime}, \frac{1}{\beta}\right) .
$$

A family of ROCs for the ABX task based on the difference model can be generated by using Equations 3, 5, and 7. Figure 2 illustrates the ROCs for four values of $d^{\prime}$ : $0.5,1.0,2.0$, and 3.0. The ROCs have been plotted in the unit square and on inverse-normal coordinates. They are symmetrical about the negative diagonal of the unit square and are almost linear on inverse-normal coordinates. A slight departure from linearity is highlighted by the dotted lines of slope one that have been positioned to pass through the coordinates of an unbiased observer.

These ROCs indicate that the quantity $z(H)-z(F)$ is almost constant for any particular value of $d^{\prime}$. Macmillan and Creelman (1991, Appendix A5.3) have provided a table that gives a good approximation to the value of $d^{\prime}$ from a particular value of $z(H)-z(F)$.

\section{The Independent-Observations Decision Strategy}

We will begin our account of the independent-observations decision strategy by reconsidering the example given earlier. The judge is presented with the heights of three children: 100,125 , and $114 \mathrm{~cm}$. To employ the independent-observations strategy, the judge initially compares the heights of the first two children. Given that the first child is shorter and the fact that the average height of the children in Room $8(110 \mathrm{~cm})$ is less than that for those in Room $10(120 \mathrm{~cm})$, the first two children are identified as coming from Rooms 8 and 10, respectively. The judge then independently assigns the third child to a room. If $115 \mathrm{~cm}$ (the mean height for both classes) is used as the criterion, the judge decides that the third child comes from Room 8 . This is the same room as that to which the first child was assigned, and thus the final decision is made: The third child comes from the same room as the first child.

From this example, we can see that, for the independentobservations strategy, the observer makes use of two independent sources of evidence when making a decision. The first source concerns the two sample stimuli presented in A and B. The difference between the evidence 
stemming from each observation can be used to make a judgment about the identity of each of the two stimuli. This corresponds to a two-alternative forced-choice task. The second source concerns only the focus stimulus. It is clear that the focus can stem from either $\mathrm{S}_{1}$ or $\mathrm{S}_{2}$, and a judgment about the identity of the focus stimulus amounts to a yes/no task. The overall ABX decision made by the observer is determined by the joint likelihood from the twocomponent subtasks within the ABX task. For an unbiased observer, who adopts a likelihood-ratio criterion of $\beta=1$, the joint likelihood is identical to adopting an unbiased criterion in each of the subtasks. However, for other criteria in the $\mathrm{ABX}$ task, the likelihood ratio is not the same as the likelihood ratios in the two subtasks.

The decision space for the independent-observations strategy consists of the joint distributions of the two independent sources of evidence available on a trial. One of the marginal distributions corresponds to the difference between $\mathrm{A}$ and $\mathrm{B}$ and, therefore, is a normal distribution with a variance of two. The second marginal distribution corresponds to the distribution of evidence in $\mathrm{X}$ and is, therefore, a standard normal distribution. Thus, the bivariate distribution of the evidence available from a single trial is given by

$$
f_{\mu_{X_{1}}, \mu_{X_{2}}}=\frac{1}{\sqrt{8} \pi} e^{-\frac{1}{4}\left(\left(X_{1}-\mu_{X_{1}}\right)^{2}+2\left(X_{2}-\mu_{X_{2}}\right)^{2}\right)},
$$

where $X_{1}$ is the difference between the evidence arising from $\mathrm{A}$ and that arising from $\mathrm{B}$ and $X_{2}$ is the evidence arising from $\mathrm{X}$. The mean of $X_{1}$ can take on the values $-d^{\prime}$ and $d^{\prime}$, whereas the mean of $X_{2}$ can take on the values zero and $d^{\prime}$.

Adopting the same definition of hits and false alarms as that for the difference strategy, the likelihood-ratio criterion for the independent-observations strategy can be calculated:

$$
\beta\left(X_{1}, X_{2}, d^{\prime}\right)=\frac{f_{d^{\prime}, d^{\prime}}+f_{-d^{\prime}, 0}}{f_{-d^{\prime}, d^{\prime}}+f_{d^{\prime}, 0}}=\frac{e^{d^{\prime}\left(X_{1}+X_{2}\right)}+e^{\frac{1}{2} d^{\prime 2}}}{e^{d^{\prime} X_{1}+\frac{1}{2} d^{\prime 2}}+e^{d^{\prime} X_{2}}} .
$$

Equations 8 and 9 define the decision space for the independent-observations strategy. This decision space is illustrated in Figure 3, which is based on a similar figure by Macmillan and Creelman (1991, Figure 7.1, p. 165). The abscissa of Figure 3 has been scaled by $1 / \sqrt{2}$ so that the standard deviations on each axis are the same. The thick lines represent various criterial boundaries. There are three separate pairs of boundaries (including the horizontal and vertical intersecting lines), each defined by a particular value of $\beta(0.5,1.0$, or 2.0$)$ and the same value of $d^{\prime}(2.0)$.

Reasoning that is similar to that used for the difference model leads to the equations for the false alarm rate and the hit rate. Again, two equations are required for each rate. However, scrutiny of the decision space in Figure 3 indicates that the same symmetry relationships hold for the independent-observations model as for the difference model. Thus, only the equations for $\beta \geq 1$ are really required, if used in conjunction with the symmetry rule of Equation 7. These two equations are

$$
\begin{aligned}
& \text { if } \beta \geq 1, F=\int_{\ln \left(\frac{\beta}{d^{\prime}}\right)+\frac{d^{\prime}}{2}}^{\infty} \int_{X_{1}\left(\beta, X_{2}, d^{\prime}\right)}^{\infty} f_{-d^{\prime}, d^{\prime}}+f_{d^{\prime}, 0} d X_{1} d X_{2}, \\
& \text { if } \beta \geq 1, \quad H=\int_{\ln \left(\frac{\beta}{d^{\prime}}\right)+\frac{d^{\prime}}{2}}^{\infty} \int_{X_{1}\left(\beta, X_{2}, d^{\prime}\right)}^{\infty} f_{-d, 0}+f_{d^{\prime}, d^{\prime}} d X_{1} d X_{2} .
\end{aligned}
$$

The lower limit of integration in the outer integrals is equal to the limit of $X_{2}\left(\beta, X_{1}, d^{\prime}\right)$ as $X_{1}$ approaches infinity.

A family of ROC curves based on Equations 10, 11, and 7 is illustrated in Figure 4. There are four curves based on the following values of $d^{\prime}: 0.5,1.0,2.0$, and 3.0. As for the difference model, they are plotted both in the unit square and on inverse-normal coordinates. For all intents, they look very similar to those obtained from the difference model. There are tables that can be used to capitalize on the fact that the ROCs are almost linear on inverse-normal coordinates, which indicates that $z(H)-$ $z(F)$ is almost the same for any particular value of $d^{\prime}$ (Kaplan, Macmillan, \& Creelman, 1978; Macmillan \& Creelman, 1991, Appendix A5.3).

\section{Modeling Data Obtained From the ABX Task}

Now that adequate models have been outlined to describe the performance of an observer in an ABX task, the issue is how best to determine the decision strategy adopted by the observer. Unlike the same-different task, in which the shape of the ROC curve is strikingly different for each decision model (Noreen, 1981), the ROCs for the $\mathrm{ABX}$ task appear almost indistinguishable. So, tests of the difference between fit statistics for the two decision models will not be productive in the case of the ABX task.

What is clearly different between the two models is the nature of the decision space. Unfortunately, in most situations, the decision space used by an observer is not itself observable, since internal noise cannot be quantified on a trial-by-trial basis. One practical solution to this problem is for the experimenter to add external noise, with known characteristics, that is sufficiently variable to swamp any internal noise. On any trial, the amount of noise added to the stimulus is known, and hence, the evidence on which the observer makes a judgment is also known. This evidence can then be plotted in a decision space, together with the actual decision made by the observer. The nature of the contours that separate different decision regions in the decision space can then be used to indicate the decision model adopted by the observer. Irwin and Hautus (1996) effectively used this approach to examine the decision model adopted by observers in a same-different task. 


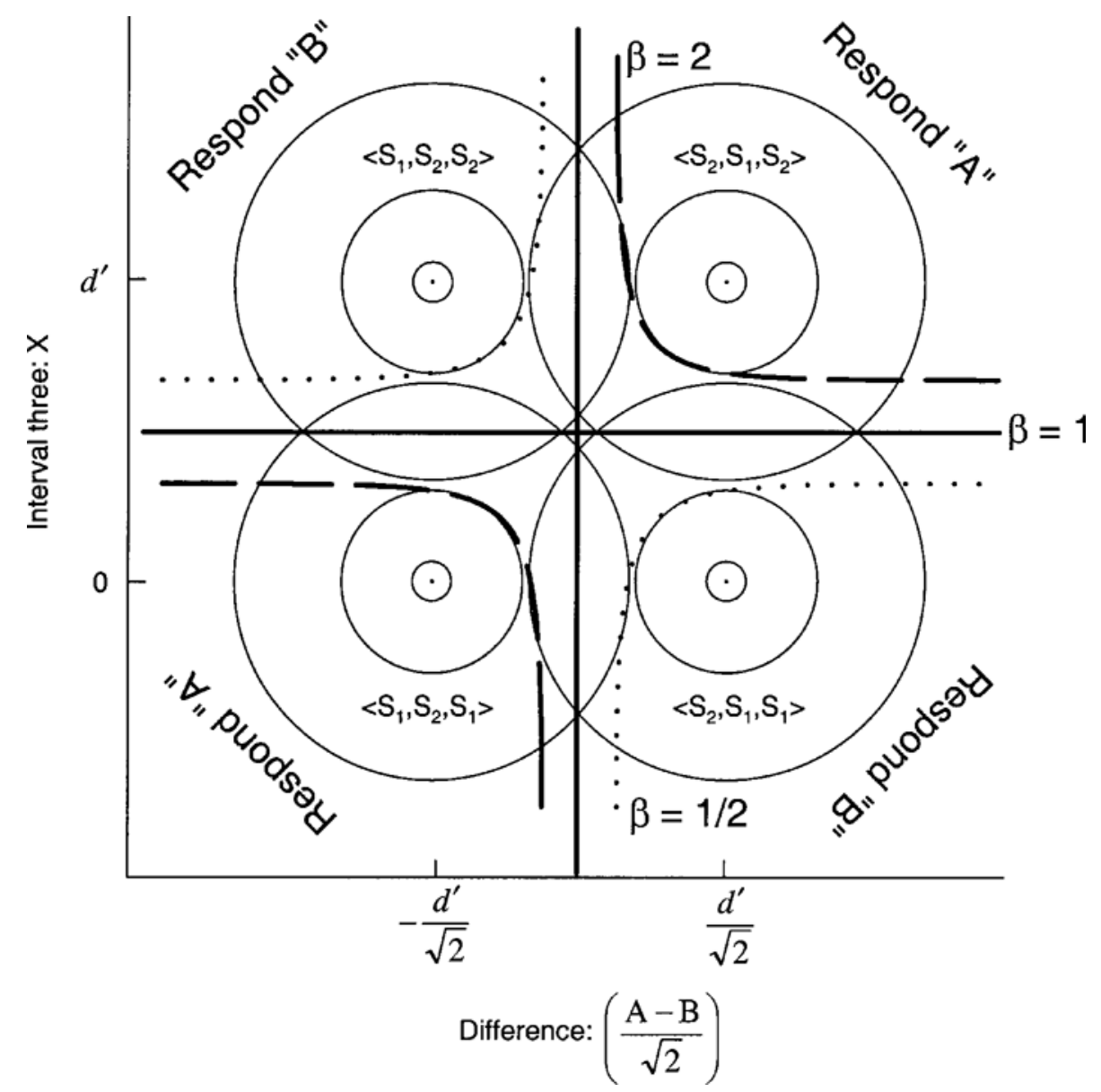

Figure 3. The decision space for the independent-observations strategy in the ABX task. The axes correspond to the two sources of independent information available on a trial: (1) the difference between the observations $A$ and $B$, which can be considered a two-alternative forced-choice subtask, and (2) the evidence available from observation $X$, which can be considered a yes/no subtask. The circles illustrate contours of equal likelihood for the distributions of evidence arising from the four stimulus sequences. The abscissa has been scaled by $1 / \sqrt{2}$ to equate the variation on each dimension. The heavy lines are criterial boundaries that divide the decision space into different response regions. The depicted boundaries correspond to likelihood ratios of 0.5 (dotted), 1.0 (solid), and 2 (dashed).

To illustrate the application of this method to the ABX task and thereby determine the decision model adopted by observers in this task, we conducted three ABX experiments. Each experiment made use of a different stimulus type: (1) circles that could vary in size, (2) visually presented numbers that could vary in value, and (3) $1-\mathrm{kHz}$ tones that could vary in amplitude. Circle and number stimuli were chosen on the basis of previous research involving the same-different task (Irwin \& Hautus, 1996). That research suggests that observers use the difference strategy for circle discrimination and the independentobservations strategy for number discrimination. Observations made within a trial for the circle and number stimuli are separated spatially; the tonal stimuli were chosen because the separation of the observations for this stimulus type is temporal.

\section{EXPERIMENT 1}

\section{Method}

Observers. Six observers participated in this experiment. Three were male, and the average age of all the participants was 31 years (range, 25-40 years). Four of the participants were naive of theories about decision strategies and psychophysical procedures $(\mathrm{O} 2$, $\mathrm{O} 3, \mathrm{O} 4$, and $\mathrm{O} 6$ ). However, $\mathrm{O} 2$ and $\mathrm{O} 3$ had participated in a pilot study and could be considered well practiced at this particular task. The remaining two observers (O1 and O5) had extensive experience on numerous different psychophysical tasks.

Stimuli and Apparatus. Individual stimuli consisted of white circular outlines presented on a $35-\mathrm{cm}$ computer monitor. The diameter of each circle was sampled from a Gaussian distribution, with parameters (mean and standard deviation) chosen to control the difficulty of the task. All of the stimuli arising from a particular distribution constituted a stimulus class. The parameters for each stimulus class were chosen so that the standardized distance between 


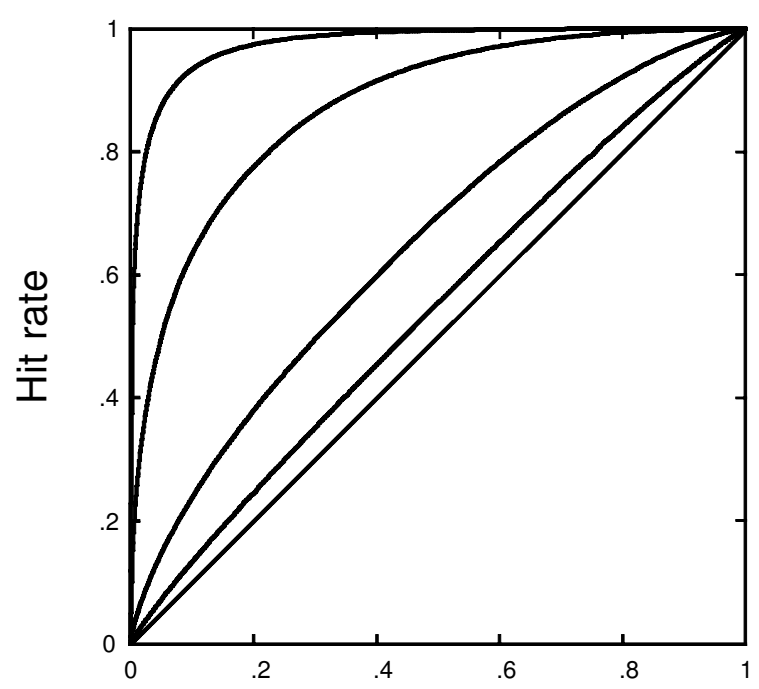

False-alarm rate

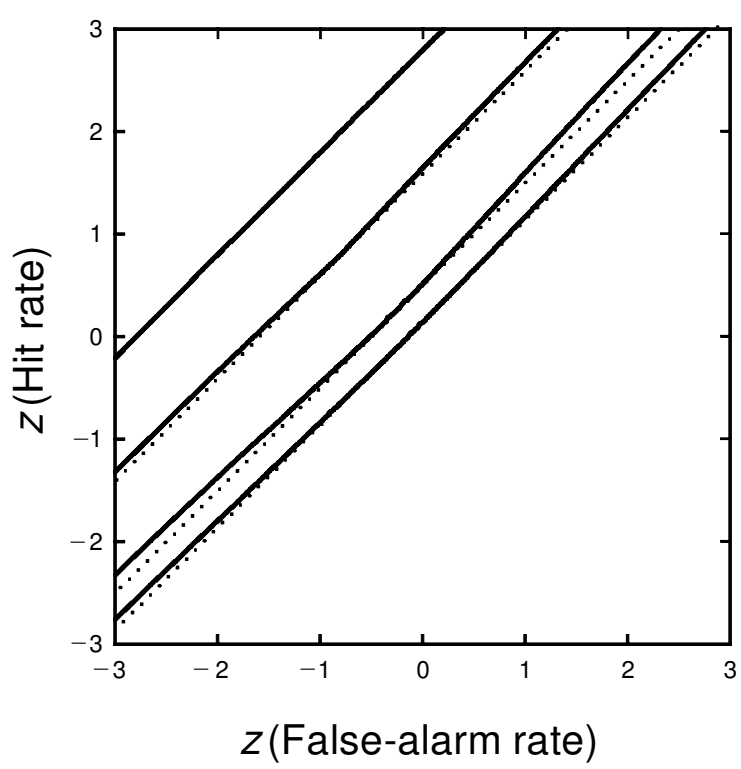

Figure 4. Theoretical receiver-operating characteristics (ROCs) for the independent-observations strategy in the ABX task. The ROCs are illustrated in the unit square (left panel) and on inverse-normal coordinates (right panel). Values of $d^{\prime}$ are 0.5 , 1.0, 2.0, and 3.0. Lines of unit slope (dotted) have been added to the right panel to highlight the slight deviation of the ROCs from linearity.

the means was one, two, or three. Fixing the standard deviation of all the stimulus classes at $8 \mathrm{~mm}$ and maintaining the midpoint between the means of any two classes to be discriminated at $38 \mathrm{~mm}$ accomplished this. Table 1 lists the mean circle diameter for each class and the standardized distance between those means.

Near the bottom of the screen was a panel that contained six response buttons, numbered consecutively from one to six. The lefthand button was also labeled certain left, and the right-hand button certain right. A third label situated above buttons three and four read uncertain .

Procedure. Before the commencement of each block of trials, two prototype stimuli were presented side by side on the screen. Their diameters were equal to the mean diameter of the $S_{1}$ and $S_{2}$ stimulus sets. The observer could look at these stimuli for as long as desired before starting the trials. These prototypes could be viewed only at the start of each block of 100 trials.

On each trial, three stimuli were presented on the monitor. One stimulus drawn from $S_{1}$ and another from $S_{2}$ were presented as standards in the upper-left (A) and upper-right (B) regions of the screen. The sides on which $S_{1}$ and $S_{2}$ were presented were chosen randomly with a probability of .5 . The third stimulus, or focus, was drawn from either $S_{1}$ or $S_{2}$, also with a probability of .5. The focus was presented in the lower-middle region $(\mathrm{X})$ of the screen.

The task of the observer was to judge which of the two standard stimuli was sampled from the same stimulus set as the focus and then to indicate their level of certainty about this judgment by selecting an appropriate response button with the computer's pointing device. Feedback was provided for $750 \mathrm{msec}$ after a response was registered. All three stimuli remained visible until the observer made a response. The screen was then cleared, and the next trial commenced $750 \mathrm{msec}$ later.

Each observer undertook 400 trials at each level of difficulty, making a total of 1,200 trials per observer. Considerable time was allowed for rest between each block of trials, and the completion of all the blocks spanned several days. The order of completion of the blocks was counterbalanced for level of difficulty. Also, each observer undertook at least 700 practice trials at various levels of difficulty before data collection began.

\section{Results and Discussion}

ROC curves fitted to the rating data were used to assess the performance of each observer. Sensitivity and bias parameters $\left(d^{\prime}\right.$ and $\beta$ ) for both the difference and the independent-observations strategies were fitted, using an iterative algorithm with chi-square as the fit statistic.

Table 2 gives the estimated sensitivity for each observer and level of difficulty. An observer who makes use of all of the information available in the stimuli would be an ideal observer. In this experiment, the estimate of sensitivity obtained for the observers should be close to the value that would be obtained by an ideal observer. This is because the major factor that limits the performance of the observers is the exogenous noise imposed by the experimenters. The performance of an ideal observer on this task can be calculated by taking the difference between the mean circle diameters for $S_{1}$ and $S_{2}$ and then dividing by the common standard deviation of both distributions. It is clear that some of the observers were performing close to ideal at all levels of difficulty (e.g., O1, O3, and O4), whereas the others were not as consistent at extracting the relevant information from the stimuli.

Table 1

Mean Circle Diameters for the $S_{1}$ and $S_{2}$ Classes for

Each of the Three Levels of Difficulty Employed in the Circle-Size Discrimination Task of Experiment 1

\begin{tabular}{|c|c|c|c|}
\hline \multirow[b]{2}{*}{ Ideal-Observer $d^{\prime}$} & \multicolumn{2}{|c|}{ Mean Diameter (mm) } & \multirow{2}{*}{$\begin{array}{c}\text { Standard } \\
\text { Deviation }(\mathrm{mm})\end{array}$} \\
\hline & $\mathrm{S}_{1}$ & $\mathrm{~S}_{2}$ & \\
\hline 0.5 & 36 & 40 & 8 \\
\hline 1.0 & 34 & 42 & 8 \\
\hline 2.0 & 30 & 46 & 8 \\
\hline
\end{tabular}

Note-The variation of circles sizes is fixed at $8 \mathrm{~mm}$ for all classes. 
Table 2

Estimates of Sensitivity $\left(d^{\prime}\right)$ Based on Each of the Two Decision

Strategies for Each Observer at Each Level of Difficulty in the

Circle Discrimination Task of Experiment 1, Fit Statistics $\left(\chi^{2}\right)$ for

Each Fitted Receiver-Operating Characteristic Curve $(d f=4)$, and Cumulative $\chi^{2}$ for Each Level of Difficulty $(d f=24)$

\begin{tabular}{|c|c|c|c|c|c|}
\hline \multirow[b]{3}{*}{ Ideal-Observer $d^{\prime}$} & \multirow[b]{3}{*}{ Observer } & \multicolumn{4}{|c|}{ Decision Model } \\
\hline & & \multicolumn{2}{|c|}{ Difference } & \multicolumn{2}{|c|}{ Independent Observation } \\
\hline & & $d^{\prime}$ & $\chi^{2}$ & $d^{\prime}$ & $\chi^{2}$ \\
\hline \multirow[t]{7}{*}{0.5} & $\mathrm{O} 1$ & 0.49 & 7.1 & 0.44 & 7.0 \\
\hline & $\mathrm{O} 2$ & 0.42 & 6.5 & 0.47 & 6.7 \\
\hline & $\mathrm{O} 3$ & 0.56 & 3.1 & 0.50 & 3.1 \\
\hline & $\mathrm{O} 4$ & 0.70 & 6.1 & 0.63 & 6.1 \\
\hline & O5 & 0.05 & 2.1 & 0.18 & 2.3 \\
\hline & O6 & 0.56 & 5.9 & 0.64 & 7.0 \\
\hline & & $\bar{d}^{\prime}=0.46$ & $\Sigma \chi^{2}=30.8$ & $\bar{d}^{\prime}=0.37$ & $\Sigma \chi^{2}=32.2$ \\
\hline \multirow[t]{7}{*}{1.0} & $\mathrm{O} 1$ & 1.01 & 1.6 & 0.91 & 1.6 \\
\hline & $\mathrm{O} 2$ & 0.79 & $11.6 *$ & 0.71 & $11.7 *$ \\
\hline & $\mathrm{O} 3$ & 1.02 & 2.3 & 0.98 & 2.6 \\
\hline & $\mathrm{O} 4$ & 1.05 & 5.5 & 0.94 & 5.5 \\
\hline & O5 & 0.92 & 4.4 & 0.82 & 4.5 \\
\hline & O6 & 0.58 & 2.5 & 0.54 & 2.5 \\
\hline & & $\bar{d}^{\prime}=0.90$ & $\Sigma \chi^{2}=27.9$ & $\bar{d}^{\prime}=0.82$ & $\Sigma \chi^{2}=28.4$ \\
\hline \multirow[t]{7}{*}{2.0} & $\mathrm{O} 1$ & 1.94 & 8.1 & 1.71 & 8.3 \\
\hline & $\mathrm{O} 2$ & 1.61 & $23.3 *$ & 1.42 & $23.7 *$ \\
\hline & $\mathrm{O} 3$ & 1.78 & $10.5^{*}$ & 1.56 & $10.2^{*}$ \\
\hline & O4 & 1.67 & 2.3 & 1.48 & 2.3 \\
\hline & O5 & 1.58 & $9.9 *$ & 1.40 & $10.2 *$ \\
\hline & O6 & -1.82 & 1.7 & -1.61 & 1.7 \\
\hline & & $\bar{d}^{\prime}=1.73$ & $\Sigma \chi^{2}=55.8^{*}$ & $\bar{d}^{\prime}=1.53$ & $\Sigma \chi^{2}=56.3^{*}$ \\
\hline
\end{tabular}

$* p<.05$.

Also provided in Table 2 are the chi-square goodnessof-fit statistics for each fitted ROC curve. The data associated with eight of the fitted ROCs had a probability of less than .05 of arising from the model. ${ }^{4}$ It is important to note how similar the chi-square values are for the two different models. The chi-squares obtained by cumulating values across observers $\left(\Sigma \chi^{2}\right)$ are also almost identical for the two models. This demonstrates fairly convincingly that any attempt to determine the decision strategy adopted by an observer in this task by using the shape of the ROC curve will be fruitless. We therefore drop this approach.

The best-fitting criterial boundaries, based on the difference and independent-observationsmodels, were determined for each decision space. The percentage of correct classifications (pcc) was employed as the goodness-offit statistic used to fit each separate boundary between each rating category. This statistic (pcc) indicates the percentage of judgments made by the observer that can be correctly predicted by the best-fitting criterial boundary. Although pcc is calculated for each individual boundary, a more useful summary statistic is provided by calculating the overall value of pcc for all of the boundaries on a particular decision space.

Table 3 lists the fit statistics for all of the observers. Also provided is the $95 \%$ confidence interval on the difference between the fit statistics obtained for each decision model. These intervals were calculated by using a bias-corrected and adjusted $\left(\mathrm{B}_{\mathrm{CA}}\right)$ bootstrap technique
(Hjorth, 1994, Section 6.6; Shao \& Tu, 1995, Chap. 4). This technique can accommodate asymmetrical sampling distributions, which lead to asymmetrical confidence intervals. The key point to note from the intervals in Table 3 is whether zero falls within the interval. This would indicate that there is no significant difference (at $\alpha=.05$ ) between the abilities of the two models to account for the data. For 13 of the 18 cases, zero does not fall within the confidence interval. Consistent with this result is the finding that, for all but 1 case, the difference model leads to a higher value of pcc than does the independentobservations model. In combination, these results provide a solid indication that the difference decision model provides a significantly better account of the data than does the independent-observations model.

For a more fine-grained analysis of the data, we consider a single observer $(\mathrm{O} 1)$ for the case in which the value of $d^{\prime}$ obtained by an ideal observer would equal one. Although this observer had participated in previous research on the ABX task, as well as on other psychophysical tasks, his results were not substantively different from those of the other observers. However, this observer did demonstrate a more stable pattern of respondingpossibly as a consequence of his extensive experience as a psychophysical observer. This makes his data ideal for illustrative purposes.

Figures 5 and 6 illustrate the best-fitting boundaries for $\mathrm{O} 1$ on the difference and independent-observations decision spaces. Each boundary is illustrated in a separate 
Table 3

The Percentage of Correct Categorizations (pcc) Made by the

Best-Fitting Decision Boundaries Based on the Difference and

Independent-Observations Models for Each Observer at Each Level of Difficulty in the Circle Discrimination task of Experiment 1

\begin{tabular}{|c|c|c|c|c|}
\hline \multirow[b]{2}{*}{ Ideal-Observer $d^{\prime}$} & \multirow[b]{2}{*}{ Observer } & \multicolumn{2}{|r|}{ pcc } & \multirow{2}{*}{$\begin{array}{c}\mathrm{B}_{\mathrm{CA}} 95 \% \\
\text { Confidence Interval }\end{array}$} \\
\hline & & Difference & Independent Observations & \\
\hline \multirow[t]{6}{*}{0.5} & $\mathrm{O} 1$ & 94.00 & 90.00 & $2.95 \leq 4.00 \leq 5.25^{*}$ \\
\hline & $\mathrm{O} 2$ & 93.80 & 89.15 & $3.60 \leq 4.65 \leq 5.95^{*}$ \\
\hline & $\mathrm{O} 3$ & 80.25 & 79.75 & $-1.25 \leq 0.50 \leq 2.10$ \\
\hline & $\mathrm{O} 4$ & 90.90 & 88.40 & $1.65 \leq 2.50 \leq 3.65^{*}$ \\
\hline & O5 & 82.95 & 82.10 & $-0.65 \leq 0.85 \leq 2.25$ \\
\hline & O6 & 93.65 & 89.45 & $3.10 \leq 4.20 \leq 5.60^{*}$ \\
\hline \multirow[t]{6}{*}{1.0} & $\mathrm{O} 1$ & 93.75 & 88.55 & $3.90 \leq 5.20 \leq 6.65^{*}$ \\
\hline & $\mathrm{O} 2$ & 84.05 & 80.85 & $1.70 \leq 3.20 \leq 4.45^{*}$ \\
\hline & $\mathrm{O} 3$ & 85.75 & 84.00 & $0.75 \leq 1.75 \leq 2.95^{*}$ \\
\hline & $\mathrm{O} 4$ & 89.35 & 87.90 & $-0.30 \leq 1.45 \leq 2.65$ \\
\hline & O5 & 86.45 & 84.95 & $0.40 \leq 1.50 \leq 2.85^{*}$ \\
\hline & O6 & 94.10 & 89.60 & $3.40 \leq 4.50 \leq 5.75^{*}$ \\
\hline \multirow[t]{6}{*}{2.0} & $\mathrm{O} 1$ & 93.60 & 89.30 & $2.85 \leq 4.30 \leq 5.65^{*}$ \\
\hline & $\mathrm{O} 2$ & 84.60 & 82.30 & $1.40 \leq 2.30 \leq 4.05^{*}$ \\
\hline & $\mathrm{O} 3$ & 89.65 & 84.90 & $3.65 \leq 4.75 \leq 6.35 *$ \\
\hline & $\mathrm{O} 4$ & 88.20 & 87.85 & $-1.00 \leq 0.35 \leq 1.75$ \\
\hline & O5 & 87.55 & 86.70 & $-0.50 \leq 0.85 \leq 2.10$ \\
\hline & O6 & 92.95 & 88.30 & $3.25 \leq 4.65 \leq 6.25^{*}$ \\
\hline
\end{tabular}

Note-Bias corrected and adjusted $\left(\mathrm{B}_{\mathrm{CA}}\right) 95 \%$ confidence intervals on the difference between the fit statistics obtained for each model are also provided. If zero lies within the interval, the two fit statistics are not significantly different at the $5 \%$ level of significance. $* p<.05$.

panel so that the error in classification made by the boundary can be observed. The pcc is included in each panel, and the overall value of pcc for each decision model is presented in the top panel. The key, located in the top-left corner of each panel, indicates the relation between the symbols on that panel and the rating scale used by the observer. In the top-left panel, the open symbols represent the trials on which the observer gave a rating of one, and the crossed symbols represent trials on which any other rating was made. Thus, the best-fitting boundary on this panel separates the rating of one from all the other ratings.

The values of pcc for each model are the most similar for the extreme criteria, whereas the greatest discrepancy, pcc $=93.3$ and 81.3 , occurs for the boundary between the ratings of three and four (the second panel on the left in each figure). In this case, for both models, the best-fitting boundary differs only slightly from that of an unbiased observer. Because such boundaries pass through the densest region of the decision space, they provide the greatest opportunity to detect inconsistencies between the models and the data.

Table 4 gives the best-fitting likelihood-ratio criterion $(\beta)$ for each of the five decision boundaries on each decision space. The criteria for the three levels of difficulty have been grouped together for each observer, to allow easy comparison. Extreme criterion values $(0.05>\beta>20)$ typically correspond to cases in which the best fit is provided by a boundary for which all of the observations lie to one side. The criteria associated with the boundary between ratings of three and four are all close to being unbiased (i.e., $\beta \approx 1$ ). This indicates that the observers assigned responses to each half of the rating scale in an unbiased way. The symmetry of the scale is probably related to this phenomenon. A second point is that, for all but three cases, the criteria for each boundary become more extreme as the difficulty of the task is increased. There are two potential causes of this effect. The observers may be varying their criteria as a function of the difficulty of the task. Alternatively, the observers may not be basing their decisions on a likelihood-ratiocriterion. Rather, they are basing their decisions on some other index of bias that is invariant as task difficulty increases.

\section{EXPERIMENT 2}

\section{Method}

Observers. All of the observers who participated in Experiment 1 also participated in this experiment.

Stimuli, Apparatus, and Procedure. The stimuli were two- or three-digit numbers presented on a computer monitor at a sufficient size to be clearly legible. Each number was sampled from a Gaussian distribution, and all of the stimuli that could be drawn from a single distribution constituted a stimulus class. The mean and variance of each class were chosen to control the difficulty of the task. The standardized difference between the means of the stimulus classes was set at half, one, or two. To accomplish this, the same standard deviation was maintained for all the classes $(\sigma=20)$, and the midpoint between the means of any two classes to be discriminated was fixed at 100. Table 5 gives the mean value for each stimulus class and the standardized difference between the means.

The same apparatus and procedure were employed as those in Experiment 1 . However, rather than circles being presented on the monitor, numbers were presented. The layout of the computer screen and the stimulus presentation format and timing were also identical, as were the response alternatives. Each observer undertook at least 700 practice trials before data collection began. A further 1,200 experimental trials were then conducted, with 400 at each level of difficulty.

\section{Results and Discussion}

Table 6 contains estimates of $d^{\prime}$ obtained from the best-fitting ROC curves for each observer at each level 


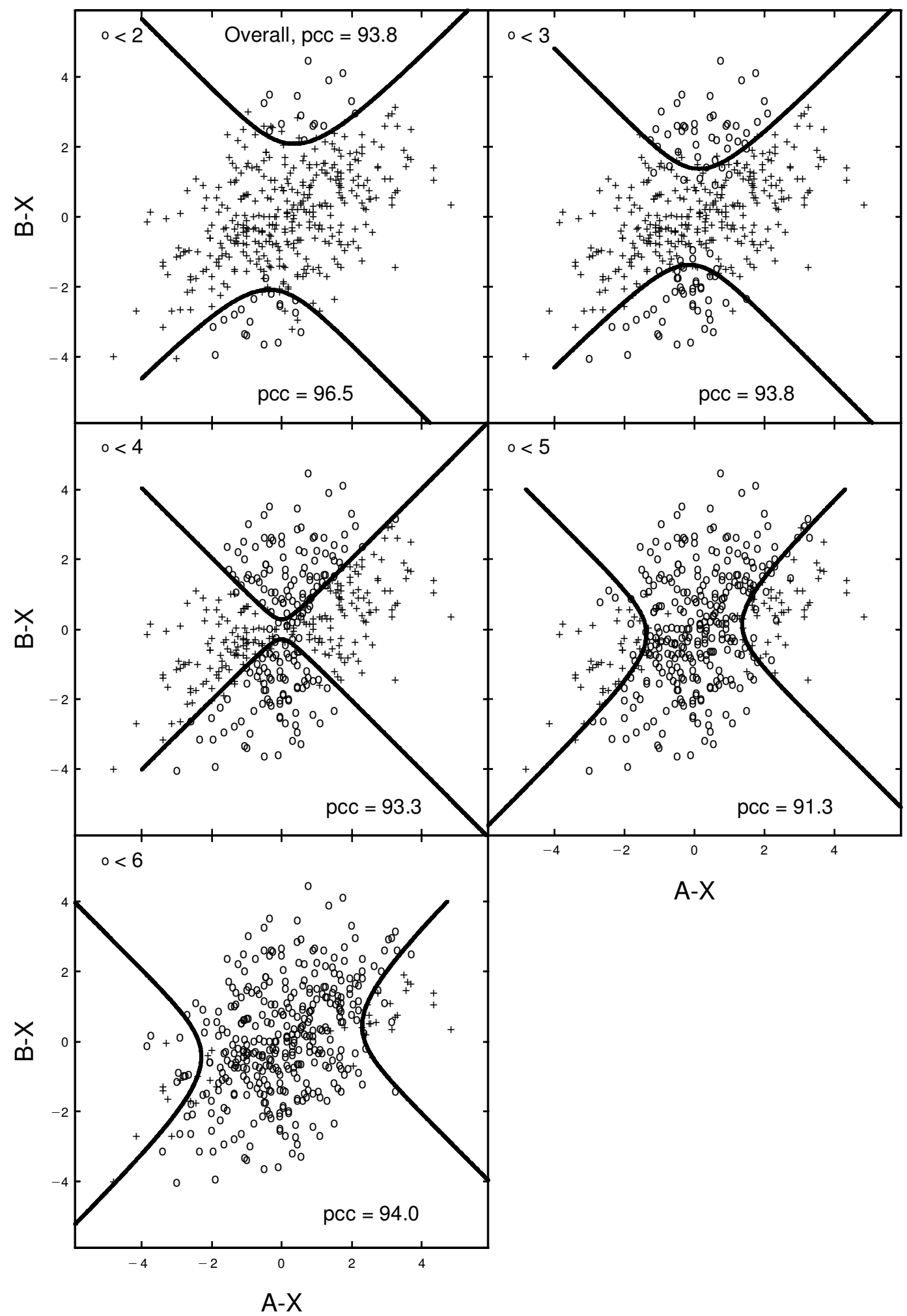

Figure 5. The decision space, based on the differencing strategy, for $\mathrm{O1}$ at the intermediate level of difficulty in the circle discrimination task of Experiment 1. Each panel illustrates the responses of the observer determined by a different criterial boundary that corresponds to a specific rating response (see the top left of each panel for details). For example, in the top-left panel, open circles correspond to a rating of one (certain the focus was a match to $A$ ), and crosses correspond to all other ratings. The heavy curves are the best-fitting criterial boundaries, and the associated fit statistic is shown in the lower right of each panel. The overall fit statistic is also provided. 


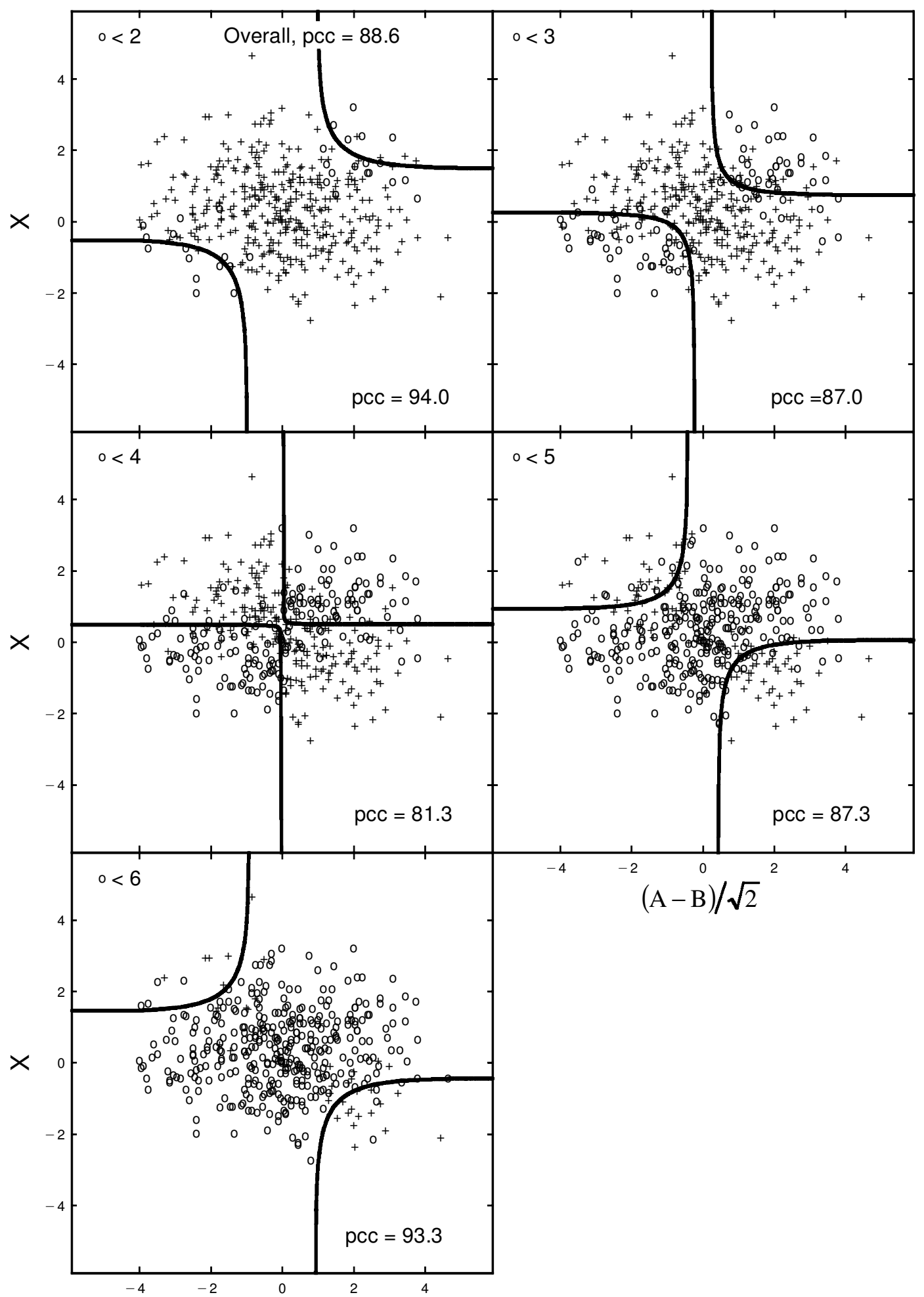

Figure 6. The decision space, based on the independent-observations strategy, for $\mathrm{O1}$ at the intermediate level of difficulty in the circle discrimination task of Experiment 1. Each panel illustrates the responses of the observer determined by a different criterial boundary that corresponds to a specific rating response (see the top left of each panel for details). For example, in the top-left panel, open circles correspond to a rating of one (certain the focus was a match to $A$ ), and crosses correspond to all other ratings. The heavy curves are the best-fitting criterial boundaries, and the associated fit statistic is shown in the lower right of each panel. The overall fit statistic is also provided. 
Table 4

The Best-Fitting Criterial Boundaries $(\beta)$, Based on the Decision-Space Analysis of Experiment 1, for Each Observer at Each Level of Difficulty

\begin{tabular}{|c|c|c|c|c|c|c|c|c|c|c|c|}
\hline \multirow[b]{2}{*}{ Observer } & \multirow{2}{*}{$\begin{array}{c}\text { Ideal- } \\
\text { Observer } d^{\prime}\end{array}$} & \multicolumn{5}{|c|}{$\begin{array}{c}\text { Difference Strategy } \\
\text { Criteria }(\beta) \text { Between Ratings }\end{array}$} & \multicolumn{5}{|c|}{$\begin{array}{c}\text { Independent-Observations Strategy } \\
\text { Criteria }(\beta) \text { Between Ratings }\end{array}$} \\
\hline & & $1-2$ & $2-3$ & $3-4$ & $4-5$ & $5-6$ & $1-2$ & $2-3$ & $3-4$ & $4-5$ & $5-6$ \\
\hline \multirow[t]{3}{*}{$\mathrm{O} 1$} & 0.5 & 1.35 & 1.16 & 1.00 & 0.91 & 0.77 & 1.45 & 1.19 & 1.01 & 0.86 & 0.75 \\
\hline & 1.0 & 1.72 & 1.32 & 1.01 & 0.76 & 0.52 & 2.70 & 1.28 & 1.01 & 0.65 & 0.40 \\
\hline & 2.0 & 5.09 & 1.61 & 1.22 & 0.55 & 0.29 & 16.4 & 2.00 & 1.10 & 0.41 & 0.22 \\
\hline \multirow[t]{3}{*}{$\mathrm{O} 2$} & 0.5 & 1.25 & 1.11 & 1.00 & 0.93 & 0.78 & 1.37 & 1.15 & 1.00 & 0.81 & 0.79 \\
\hline & 1.0 & 2.27 & 2.27 & 1.02 & 0.71 & 0.43 & 4.76 & 3.23 & 0.87 & 0.31 & 0.18 \\
\hline & 2.0 & 15.4 & 3.70 & 0.97 & 0.46 & 0.03 & 62.9 & 5.56 & 0.83 & 0.75 & 0.01 \\
\hline \multirow[t]{3}{*}{$\mathrm{O} 3$} & 0.5 & 1.82 & 1.06 & 1.05 & 0.98 & 0.47 & 2.04 & 1.06 & 1.03 & 1.00 & 0.46 \\
\hline & 1.0 & 2.04 & 1.96 & 1.05 & 0.70 & 0.52 & 3.23 & 1.89 & 1.10 & 0.60 & 0.35 \\
\hline & 2.0 & 4.76 & 4.55 & 0.80 & 0.40 & 0.13 & 11.1 & 7.69 & 0.56 & 0.22 & 0.07 \\
\hline \multirow[t]{3}{*}{ O4 } & 0.5 & 1.49 & 1.23 & 1.00 & 0.87 & 0.76 & 1.79 & 1.22 & 0.99 & 0.85 & 0.60 \\
\hline & 1.0 & 8.33 & 2.04 & 1.00 & 0.58 & 0.48 & 4.17 & 3.03 & 1.03 & 0.53 & 0.46 \\
\hline & 2.0 & 5.88 & 2.33 & 1.00 & 0.31 & 0.22 & 12.5 & 6.25 & 0.92 & 0.17 & 0.09 \\
\hline \multirow[t]{3}{*}{ O5 } & 0.5 & 1.64 & 1.08 & 1.00 & 0.85 & 0.56 & 1.75 & 1.22 & 0.96 & 0.79 & 0.49 \\
\hline & 1.0 & 5.26 & 1.41 & 0.92 & 0.64 & 0.14 & 7.69 & 1.82 & 1.09 & 0.55 & 0.11 \\
\hline & 2.0 & 25.3 & 1.96 & 0.91 & 0.35 & 0.05 & 26.9 & 3.33 & 0.42 & 0.21 & 0.01 \\
\hline \multirow[t]{3}{*}{ O6 } & 0.5 & 1.33 & 1.09 & 1.00 & 0.93 & 0.79 & 1.28 & 1.15 & 1.01 & 0.91 & 0.75 \\
\hline & 1.0 & 2.22 & 1.32 & 0.99 & 0.74 & 0.45 & 2.63 & 1.67 & 0.98 & 0.55 & 0.27 \\
\hline & 2.0 & 5.26 & 2.13 & 0.97 & 0.47 & 0.24 & 6.67 & 3.03 & 0.74 & 0.41 & 0.13 \\
\hline
\end{tabular}

Note-The criterial boundaries for each level of difficulty (ideal-observer $d^{\prime}=0.5,1.0$, and 2.0) have been grouped for each observer, to allow easy comparison.

of difficulty. The means of the estimates of $d^{\prime}$ indicate that performance reached that expected of an ideal observer only for the hardest level of difficulty. The cumulative chi-squares indicate that both models provided a good fit to the data for the two hardest levels of difficulty, whereas both models were rejected at the easiest level.

The best-fitting criterial boundaries, based on the difference and independent-observations models, are given in Table 7. Values of pcc for each model and the confidence interval for the difference between these values are also provided. Only 3 of the 18 cases do not yield a significant difference between the fit statistics. This again provides convincing evidence that the difference decision model provides a significantly better account of the data than does the independent-observations model. Given that the results for this experiment are highly consistent with those for Experiment 1, we do not present the decision spaces here.

Table 8 gives the best-fitting likelihood-ratiocriteria $(\beta)$ for all of the decision boundaries on each decision space. The two patterns noted in Table 4 of Experiment 1 are again present. An unbiased criterion is typically adopted for the boundary between ratings of three and four. However, in Table 8, there is reduced consistency in the observed change to a more extreme criterion as task difficulty increases. The reason for this effect is unclear, since there is little difference in the fit statistics for these two experiments.

\section{EXPERIMENT 3}

\section{Method}

Observers. Five of the 6 observers (O1-O5) who participated in the previous experiments also participated in Experiment 3. One new naive observer was also recruited $(\mathrm{O} 7)$. The average age of the participants remained 31 years (range, 23-40 years), but now 4 were male.

Stimuli. The stimuli were $1000-\mathrm{Hz}$ sinusoidal tones of $100-\mathrm{msec}$ duration that were generated digitally (Hewlett Packard, 8904A). The tones were gated electronically, using a Hanning window function with a 10-msec rise/fall time. The amplitude of each tone was sampled from a Gaussian distribution, and all of the stimuli that could be drawn from a single distribution constituted a stimulus class. The standardized difference between the means of the stimulus classes were again set at half, one, or two. The same standard deviation was maintained for each class ( $\sigma=5 \mathrm{~dB}$ SPL), and the midpoint between the means of any two classes to be discriminated was fixed at $55 \mathrm{~dB}$ SPL. Table 9 gives the mean value for each stimulus class and the standardized difference between the means.

Procedure. The observers were seated in a sound-attenuating chamber (Amplaid Model E). On a table in front of the observer was a keyboard, on which their confidence-ratings were registered, and a panel of three light-emitting diodes (LEDs) that were illuminated to indicate the observation intervals and to provide feedback to the observer.

Each trial began with a 150 -msec warning flash. After a pause of $250 \mathrm{msec}$, the three 250 - $\mathrm{msec}$ tones were presented in sequence to the observer's right ear. A pause of $500 \mathrm{msec}$ was inserted between each observation interval. An LED was illuminated during each observation interval. The first two observation intervals contained the standard stimuli-that is, one tone with an amplitude drawn from the $S_{1}$ distribution, and the other tone with an amplitude drawn from the $S_{2}$ distribution. The order of presentation of the $S_{1}$ and $S_{2}$ tones

Table 5

Mean Values for the $S_{1}$ and $S_{2}$ Classes for Each of the Three Levels of Difficulty Employed in the Number-Discrimination Task of Experiment 2

\begin{tabular}{cccc}
\hline & \multicolumn{2}{c}{ Mean Value } & \\
\cline { 2 - 3 } Ideal-Observer $d^{\prime}$ & $\mathrm{S}_{1}$ & $\mathrm{~S}_{2}$ & Standard Deviation \\
\hline 0.5 & 95 & 105 & 20 \\
1.0 & 90 & 110 & 20 \\
2.0 & 80 & 120 & 20 \\
\hline
\end{tabular}


Table 6

Estimates of $d^{\prime}$ Obtained From the Ratings Made by Observers for the Number Discrimination Task of Experiment 2

Decision Model

\begin{tabular}{cccccccc} 
& \multicolumn{3}{c}{ Difference } & & \multicolumn{3}{c}{ Independent Observations } \\
\cline { 2 - 4 } \cline { 7 - 8 } Observer & 0.5 & 1.0 & 2.0 & & 0.5 & 1.0 & 2.0 \\
\hline O1 & 0.65 & 0.85 & 1.88 & & 0.59 & 0.76 & 1.66 \\
O2 & 0.56 & 0.80 & 1.70 & & 0.50 & 0.72 & 1.50 \\
O3 & 0.20 & 0.55 & 1.76 & & 0.20 & 0.72 & 1.55 \\
O4 & 0.64 & 0.60 & 1.70 & & 0.58 & 0.54 & 1.51 \\
O5 & 0.44 & 0.86 & 1.49 & & 0.40 & 0.77 & 1.31 \\
O6 & 0.66 & 0.76 & 1.65 & & 0.81 & 0.68 & 1.49 \\
$\bar{d}^{\prime}$ & 0.53 & 0.74 & 1.69 & & 0.51 & 0.70 & 1.50 \\
$\Sigma \chi^{2}$ & 34.47 & 24.92 & $69.48^{*}$ & 36.43 & 19.96 & $69.74^{*}$ \\
\hline
\end{tabular}

Note-The ideal-observer $d^{\prime}$ 's are given for each model. The mean of the estimates of $d^{\prime}$ within each level of difficulty for each model and the cumulative chi-square statistics $(d f=24)$ are also presented. $* p<.05$.

was randomized. The third observation interval contained the focus - a tone with an amplitude drawn from either the $S_{1}$ or the $S_{2}$ distribution, with an equal chance of presentation for each.

After the stimuli were presented, the observers rated their confidence that the stimulus presented in the third interval was drawn from the same class as that presented in the first interval or the second interval. There was no time limit for making this judgment. After the observers had responded on the keyboard, feedback was provided by illuminating one of two LEDs for $100 \mathrm{msec}$. The first LED indicated that the focus was a match to the first interval, whereas the second LED indicated a match to the second interval. The next trial was initiated $300 \mathrm{msec}$ after the feedback LED was extinguished.

As for the previous two experiments, each observer undertook 400 trials at each level of difficulty. To ensure adequate training of the naive observers, each observer completed at least 1,000 practice trials before data collection began. The order of completion of blocks was counterbalanced for level of difficulty.

\section{Results and Discussion}

Table 10 contains the estimates of $d^{\prime}$ for each observer at each level of difficulty. Sensitivity appears to be similar to that in Experiments 1 and 2. The cumulative chisquare statistics in Table 10 indicate that the models provided a good fit at the two hardest levels of difficulty but were rejected at the remaining level. This has been a systematic finding over all three experiments.

The criterial boundaries that best fit the judgments made by the observers are presented in Table 11, together with goodness-of-fit statistics for each model and confidence intervals on the difference between those statistics. The results presented in Table 11 look different on a number of counts from those for the previous experiments. First, of the 18 comparisons of model performance, only 1 is significantly different. This is almost the opposite pattern to that in the previous experiments. However, a closer look at the individual values of pec indicates that the value of this statistic is higher for the difference model than for the independent-observations model for 16 of the 18 comparisons. Thus, even though the differences are not significant on a case-by-case basis, there is sufficient consistency in the direction of the difference to provide good evidence that the difference model provides the best account of the decision strategy adopted by the observers (note that a sign test yields $p<.05$ ). A second difference between the results given in Table 11 and those from the previous two experiments is in the overall magnitude of the pcc statistics-they are lower for the auditory task. Consideration of the number of good fits can illustrate this. If we consider a good fit to have a value of pcc greater than .9, there were eight, seven, and one good fits (for the better-fitting difference model) in Ex-

Table 7

Values of Percentage of Correct Categorizations (pcc)

for the Number Discrimination Task of Experiment 2

\begin{tabular}{|c|c|c|c|c|}
\hline \multirow[b]{2}{*}{ Ideal-Observer $d^{\prime}$} & \multirow[b]{2}{*}{ Observer } & \multicolumn{2}{|r|}{ pcc } & \multirow{2}{*}{$\begin{array}{c}\mathrm{B}_{\mathrm{CA}} 95 \% \\
\text { Confidence Interval }\end{array}$} \\
\hline & & Difference & Independent Observations & \\
\hline \multirow[t]{6}{*}{0.5} & $\mathrm{O} 1$ & 92.50 & 92.15 & $-1.05 \leq 0.35 \leq 1.50$ \\
\hline & $\mathrm{O} 2$ & 76.25 & 76.15 & $-0.50 \leq 0.10 \leq 0.90$ \\
\hline & $\mathrm{O} 3$ & 89.95 & 88.90 & $0.10 \leq 1.05 \leq 1.90 *$ \\
\hline & $\mathrm{O} 4$ & 90.10 & 86.85 & $2.45 \leq 3.25 \leq 4.25^{*}$ \\
\hline & O5 & 88.20 & 84.65 & $2.35 \leq 3.55 \leq 5.20^{*}$ \\
\hline & O6 & 92.10 & 88.40 & $2.70 \leq 3.70 \leq 5.00^{*}$ \\
\hline \multirow[t]{6}{*}{1.0} & $\mathrm{O} 1$ & 92.10 & 85.50 & $5.05 \leq 6.60 \leq 8.30^{*}$ \\
\hline & $\mathrm{O} 2$ & 88.05 & 85.60 & $1.25 \leq 2.45 \leq 3.85^{*}$ \\
\hline & $\mathrm{O} 3$ & 88.05 & 77.40 & $8.85 \leq 10.65 \leq 13.25^{*}$ \\
\hline & $\mathrm{O} 4$ & 87.05 & 82.30 & $3.30 \leq 4.75 \leq 6.05^{*}$ \\
\hline & O5 & 92.00 & 87.45 & $3.10 \leq 4.55 \leq 5.95^{*}$ \\
\hline & O6 & 91.40 & 88.60 & $1.75 \leq 2.80 \leq 3.95^{*}$ \\
\hline \multirow[t]{6}{*}{2.0} & $\mathrm{O} 1$ & 92.00 & 90.10 & $0.25 \leq 1.90 \leq 3.35^{*}$ \\
\hline & $\mathrm{O} 2$ & 89.30 & 86.00 & $2.15 \leq 3.30 \leq 4.60^{*}$ \\
\hline & $\mathrm{O} 3$ & 90.45 & 86.25 & $2.45 \leq 4.20 \leq 6.30^{*}$ \\
\hline & $\mathrm{O} 4$ & 85.25 & 83.70 & $-0.40 \leq 1.55 \leq 3.05$ \\
\hline & O5 & 88.00 & 85.10 & $1.40 \leq 2.90 \leq 4.45^{*}$ \\
\hline & O6 & 87.05 & 84.75 & $0.95 \leq 2.30 \leq 3.70 *$ \\
\hline
\end{tabular}

Note-Also provided are the $95 \%$ confidence intervals on the difference between the fit statistics obtained for each model. $* p<.05$. 
Table 8

The Best-Fitting Criterial Boundaries $(\beta)$, Based on the Decision-Space Analysis of Experiment 2 for Each Observer at Each Level of Difficulty

\begin{tabular}{|c|c|c|c|c|c|c|c|c|c|c|c|}
\hline \multirow[b]{3}{*}{ Observer } & \multirow[b]{3}{*}{ Ideal-Observer $d^{\prime}$} & \multicolumn{10}{|c|}{ Criteria $(\beta)$ Between Ratings } \\
\hline & & \multicolumn{5}{|c|}{ Difference Strategy } & \multicolumn{5}{|c|}{ Independent-Observations Strategy } \\
\hline & & $1-2$ & $2-3$ & $3-4$ & $4-5$ & $5-6$ & $1-2$ & $2-3$ & $3-4$ & $4-5$ & $5-6$ \\
\hline \multirow[t]{3}{*}{$\mathrm{O} 1$} & 0.5 & 1.32 & 1.06 & 1.00 & 0.95 & 0.65 & 1.28 & 1.09 & 1.00 & 0.90 & 0.75 \\
\hline & 1.0 & 1.85 & 1.22 & 0.99 & 0.81 & 0.93 & 2.08 & 1.45 & 0.97 & 0.77 & 0.47 \\
\hline & 2.0 & 2.86 & 1.37 & 0.97 & 0.57 & 0.36 & 5.56 & 1.92 & 1.08 & 0.61 & 0.14 \\
\hline \multirow[t]{3}{*}{$\mathrm{O} 2$} & 0.5 & 1.61 & 1.49 & 0.97 & 0.66 & 0.66 & 1.72 & 1.49 & 1.00 & 0.51 & 0.51 \\
\hline & 1.0 & 3.13 & 1.43 & 1.00 & 0.50 & 0.21 & 3.03 & 2.27 & 0.98 & 0.53 & 0.32 \\
\hline & 2.0 & $>100$ & 2.08 & 1.00 & 0.29 & 0.09 & $>100$ & 3.70 & 1.00 & 0.18 & 0.02 \\
\hline \multirow[t]{3}{*}{$\mathrm{O} 3$} & 0.5 & 1.85 & 1.85 & 1.00 & 0.66 & 0.65 & 1.79 & 1.79 & 1.09 & 0.49 & 0.49 \\
\hline & 1.0 & 1.45 & 1.00 & 1.00 & 1.00 & 0.17 & 4.55 & 1.02 & 1.02 & 0.60 & 0.06 \\
\hline & 2.0 & 5.26 & 1.00 & 1.00 & 1.00 & 0.03 & 16.7 & 1.10 & 1.10 & 1.00 & 0.01 \\
\hline \multirow[t]{3}{*}{$\mathrm{O} 4$} & 0.5 & 1.85 & 1.41 & 1.00 & 0.47 & 0.47 & 1.67 & 1.64 & 1.00 & 0.51 & 0.51 \\
\hline & 1.0 & 5.00 & 1.41 & 1.00 & 0.64 & 0.23 & 3.57 & 3.57 & 1.03 & 0.40 & 0.24 \\
\hline & 2.0 & 4.35 & 2.13 & 1.00 & 0.58 & 0.17 & 10.3 & 6.73 & 1.10 & 0.37 & 0.16 \\
\hline \multirow[t]{3}{*}{ O5 } & 0.5 & 1.35 & 1.04 & 1.00 & 0.97 & 0.74 & 1.33 & 1.06 & 1.00 & 0.92 & 0.74 \\
\hline & 1.0 & 2.38 & 1.32 & 1.00 & 0.79 & 0.32 & 2.94 & 1.54 & 1.01 & 0.64 & 0.32 \\
\hline & 2.0 & 12.4 & 1.22 & 1.00 & 0.80 & 0.01 & 50.3 & 2.70 & 1.00 & 0.37 & 0.02 \\
\hline \multirow[t]{3}{*}{ O6 } & 0.5 & 1.69 & 1.09 & 1.00 & 0.93 & 0.68 & 1.72 & 1.19 & 0.99 & 0.88 & 0.57 \\
\hline & 1.0 & 4.76 & 1.59 & 1.00 & 0.64 & 0.29 & 6.67 & 3.57 & 0.99 & 0.47 & 0.15 \\
\hline & 2.0 & 5.56 & 2.13 & 1.03 & 0.22 & 0.13 & 7.14 & 7.14 & 0.98 & 0.17 & 0.05 \\
\hline
\end{tabular}

Note-The criterial boundaries for each level of difficulty (ideal-observer $d^{\prime}=0.5,1.0$, and 2.0) have been grouped for each observer, to allow easy comparison.

periments $1-3$, respectively. A more formal approach, a post hoc analysis of variance using Tukey's HSD, indicates that the arcsine-transformed values of pcc obtained for Experiment 3 are significantly different from those obtained in Experiment $2(p<.05)$ and from those obtained in Experiment $1(p<.05)$, whereas those obtained in the first two experiments are not significantly different.

The best-fitting likelihood-ratio criteria $(\beta)$ are given in Table 12. The two patterns noted in the previous experiments (Tables 4 and 8 ) are again present. However, the values of $\beta$ for the boundary between ratings three and four are not as tightly clustered around one as they were in Experiments 1 and 2. In addition, there is a proliferation of more extreme values of $\beta$ (i.e., $0.05>\beta>$ $20)$. These differences may be explained by the reduced fit statistics for this experiment when compared with the previous two.

\section{GENERAL DISCUSSION}

\section{Modeling of the ROCs}

Although it is not possible to determine the decision strategy adopted by an observer from his or her ROC, it is still desirable to consider the effectiveness of these $\mathrm{ABX}$ models at accounting for the data. It is clear that the fits of the two models to the empirical ROCs are particularly good. The cumulative chi-square statistics reveal that the data do not depart significantly from the model for the two more difficult conditions in any of the experiments. However, for the easiest level of difficultywhere the value of $d^{\prime}$ that would be obtained by an ideal observer is two-the cumulative chi-square statistics indicate that the data differ significantly from the model in all three experiments. A closer look at the data indicates that, for this case, the magnitude of the cumulative chisquare statistics can be traced to the data of but a few observers. The data of individual observers differs from the best-fitting models for 3, 2, and 1 observers in Experiments 1-3, respectively. Furthermore, the same observers tended to yield poor fits across experiments. For example, the data of $\mathrm{O} 2$ differed significantly from the bestfitting models in all three experiments.

The level of performance of the observers, as assessed by the mean value of $d^{\prime}$ across all the observers within a condition, was in most cases worse than would be expected of an ideal observer. Given that the internal noise of the observers was adequately swamped by the stimulus noise, this was, at first, a surprising finding. It suggests that such factors as fatigue, response errors, and criterial drift or relocation were influencing performance to a significant degree. Previous research (Irwin \& Hautus, 1996) that used this method in conjunction with the same-different task found performance to be near ideal. That research employed experienced observers. Thus, one difference from that earlier work is that the observers were, in this instance, less experienced at participating in psychophysical experiments. Consequently, a considerable amount of procedural learning was probably taking

Table 9

Mean Sound-Pressure Levels for the $S_{1}$ and $S_{2}$ Classes for Each of the Three Levels of Difficulty Employed in the Auditory Discrimination Task of Experiment 3

\begin{tabular}{cccc}
\hline & \multicolumn{2}{c}{ Mean Level (dB SPL) } & $\begin{array}{c}\text { Standard } \\
\text { Ideal-Observer } d^{\prime}\end{array}$ \\
\cline { 2 - 4 } $\mathrm{S}_{1}$ & $\mathrm{~S}_{2}$ & Deviation (dB SPL) \\
\hline 0.5 & 53.8 & 56.3 & 5.0 \\
1.0 & 52.5 & 57.5 & 5.0 \\
2.0 & 50.0 & 60.0 & 5.0 \\
\hline
\end{tabular}


Table 10

Estimates of $d^{\prime}$ Obtained From the Ratings Made by Observers for the Auditory Amplitude Discrimination Task of Experiment 3

Decision Model

\begin{tabular}{cccccccc}
\hline & \multicolumn{5}{c}{ Decision Model } \\
\cline { 2 - 4 } Observer & 0.5 & 1.0 & 2.0 & & 0.5 & 1.0 & 2.0 \\
\cline { 2 - 4 } \cline { 6 - 7 } Ondependent Observations \\
\hline O1 & 0.49 & 0.90 & 1.66 & & 0.56 & 0.81 & 1.47 \\
O2 & 0.14 & 0.90 & 1.53 & & 0.35 & 0.81 & 1.35 \\
O3 & 0.75 & 0.57 & 1.34 & & 0.68 & 0.52 & 1.20 \\
O4 & 0.65 & 0.70 & 1.77 & & 0.58 & 0.65 & 1.57 \\
O5 & 0.09 & 0.85 & 1.00 & & 0.32 & 0.76 & 0.89 \\
O7 & 0.13 & 0.54 & 1.46 & & 0.12 & 0.48 & 1.30 \\
$\bar{d}^{\prime}$ & 0.38 & 0.74 & 1.46 & & 0.44 & 0.67 & 1.30 \\
$\Sigma$ & 24.32 & 26.90 & $41.31 *$ & 26.64 & 26.49 & $41.62 *$ \\
\hline
\end{tabular}

Note-The ideal observer $d^{\prime}$ is given for each model. The mean of the estimates of $d^{\prime}$ within each level of difficulty for each model, and the cumulative chi-square statistics $(d f=24)$ are also presented. $* p<.05$.

place, and this may have contributed to the overall lower performance in the present experiments. Another explanation for the difference in performance on the two tasks is that the cognitive load for the ABX task may be larger than that for the same-different task. Both explanations have intuitive appeal.

Although the average performance across observers was less than expected, there were several instances in which the performance of an individual observer was slightly better than ideal. This supra-ideal performance could, in part, be attributed to sampling variability. However, O4 demonstrated supra-ideal performance at the hardest level of difficulty in all three experiments (based on the difference strategy). This may hint at a minor problem with the models that we used. It is not that the observers are performing better than ideal; rather, our models are slightly overestimating their performance. Alternatively, this supra-ideal performance could be evidence in favor of the independent-observations strategy. Models based on this strategy typically estimate lower levels of performance, given the same data (refer to Tables 2, 6, and 10). For example, when the independentobservations strategy is used, the performance of $\mathrm{O} 4$ at the hardest level of difficulty is better than that of an ideal observer for only one of the three experiments.

A procedural matter that may have had an unanticipated side effect was the use of trial-by-trial feedback. (Robinson \& Watson [1972, pp. 108-110] provide a brief overview of the potential benefits of using feedback after a block of trials, rather than after every trial.) In our experiments, the nature of the stimulus classes meant that feedback could appear paradoxical to the observer. For example, in a trial in Experiment 1, the standard stimuli may have been a large circle and a small circle, and the focus stimulus an even smaller circle. A reasonable judge would say that the focus stimulus was a match to the smaller of the standard stimuli irrespective of the decision strategy employed. However, after making this response, an observer may be given feedback indicating that they made an incorrect decision. Verbal (and nonverbal) comments from observers suggested that this "paradoxical" feedback, when it occurred, was disturbing to them. This dissonance occurred despite the fact that the observers were fully briefed on the nature of the task. Although the feedback was important to assist observers in learning the task, it is also possible that "paradoxical" feedback could encourage the relocation of criteria or even an overall change in the observer's approach to the task.

Table 11

Values of Percentage of Correct Categorizations (pcc) for the Auditory Amplitude Discrimination Task of Experiment 3

\begin{tabular}{|c|c|c|c|c|}
\hline \multirow[b]{2}{*}{ Ideal-Observer $d^{\prime}$} & \multirow[b]{2}{*}{ Observer } & \multicolumn{2}{|r|}{ pcc } & \multirow{2}{*}{$\begin{array}{c}\mathrm{B}_{\mathrm{CA}} 95 \% \\
\text { Confidence Interval }\end{array}$} \\
\hline & & Difference & Independent Observations & \\
\hline \multirow[t]{6}{*}{0.5} & O1 & 89.00 & 88.05 & $-0.15 \leq 0.95 \leq 1.75$ \\
\hline & $\mathrm{O} 2$ & 83.30 & 82.75 & $-0.50 \leq 0.55 \leq 1.70$ \\
\hline & $\mathrm{O} 3$ & 86.15 & 86.00 & $-0.80 \leq 0.15 \leq 0.90$ \\
\hline & $\mathrm{O} 4$ & 89.85 & 89.90 & $-1.00 \leq-0.05 \leq 0.95$ \\
\hline & O5 & 81.50 & 81.30 & $-0.85 \leq 0.20 \leq 1.00$ \\
\hline & $\mathrm{O} 7$ & 84.30 & 83.35 & $-0.25 \leq 0.95 \leq 2.65$ \\
\hline \multirow[t]{6}{*}{1.0} & $\mathrm{O} 1$ & 87.95 & 87.75 & $-0.70 \leq 0.20 \leq 1.25$ \\
\hline & $\mathrm{O} 2$ & 86.65 & 86.00 & $-0.30 \leq 0.65 \leq 1.55$ \\
\hline & $\mathrm{O} 3$ & 88.50 & 88.30 & $-0.55 \leq 0.20 \leq 0.95$ \\
\hline & $\mathrm{O} 4$ & 91.05 & 90.35 & $-0.05 \leq 0.70 \leq 1.75$ \\
\hline & O5 & 82.25 & 82.60 & $-1.55 \leq-0.35 \leq 0.35$ \\
\hline & $\mathrm{O} 7$ & 80.25 & 79.85 & $-0.95 \leq 0.40 \leq 2.10$ \\
\hline \multirow[t]{6}{*}{2.0} & $\mathrm{O} 1$ & 88.65 & 88.50 & $-1.15 \leq 0.15 \leq 1.25$ \\
\hline & $\mathrm{O} 2$ & 85.40 & 84.90 & $-0.45 \leq 0.50 \leq 1.40$ \\
\hline & $\mathrm{O} 3$ & 83.45 & 82.90 & $-0.80 \leq 0.55 \leq 1.50$ \\
\hline & $\mathrm{O} 4$ & 89.80 & 88.75 & $0.15 \leq 1.05 \leq 2.05^{*}$ \\
\hline & O5 & 81.05 & 80.15 & $-0.15 \leq 0.90 \leq 1.95$ \\
\hline & $\mathrm{O} 7$ & 78.05 & 77.70 & $-1.05 \leq 0.35 \leq 1.80$ \\
\hline
\end{tabular}

Note-Also provided are the $95 \%$ confidence intervals on the difference between the fit statistics obtained for each model. Note that the only significant difference between the performances of the two models occurs for $\mathrm{O} 4$ in the easiest condition. $* p<.05$. 
Table 12

The Best-Fitting Criterial Boundaries $(\beta)$, Based on the Decision-Space Analysis of Experiment 3 for Each Observer at Each Level of Difficulty

\begin{tabular}{|c|c|c|c|c|c|c|c|c|c|c|c|}
\hline \multirow[b]{3}{*}{ Observer } & \multirow[b]{3}{*}{ Ideal-Observer $d^{\prime}$} & \multicolumn{10}{|c|}{ Criteria $(\beta)$ Between Ratings } \\
\hline & & \multicolumn{5}{|c|}{ Difference Strategy } & \multicolumn{5}{|c|}{ Independent-Observations Strategy } \\
\hline & & $1-2$ & $2-3$ & $3-4$ & $4-5$ & $5-6$ & $1-2$ & $2-3$ & $3-4$ & $4-5$ & $5-6$ \\
\hline \multirow[t]{3}{*}{$\mathrm{O} 1$} & 0.5 & 1.45 & 1.15 & 1.05 & 0.91 & 0.69 & 1.67 & 1.23 & 1.03 & 0.77 & 0.64 \\
\hline & 1.0 & 2.78 & 1.54 & 1.11 & 0.49 & 0.19 & 3.85 & 2.04 & 1.01 & 0.30 & 0.14 \\
\hline & 2.0 & 15.9 & 4.76 & 1.82 & 0.25 & 0.05 & 22.8 & 8.33 & 1.12 & 0.08 & 0.04 \\
\hline \multirow[t]{3}{*}{$\mathrm{O} 2$} & 0.5 & 1.37 & 1.12 & 1.06 & 0.75 & 0.69 & 1.85 & 1.18 & 1.09 & 0.71 & 0.53 \\
\hline & 1.0 & 4.00 & 1.92 & 1.05 & 0.49 & 0.22 & 4.35 & 2.04 & 1.12 & 0.30 & 0.22 \\
\hline & 2.0 & $>100$ & 4.00 & 0.93 & 0.10 & 0.01 & $>100$ & 4.35 & 0.93 & 0.14 & 0.01 \\
\hline \multirow[t]{3}{*}{ O3 } & 0.5 & 1.59 & 1.23 & 1.05 & 0.62 & 0.56 & 2.00 & 1.20 & 1.09 & 0.65 & 0.50 \\
\hline & 1.0 & 3.85 & 3.70 & 1.16 & 0.32 & 0.23 & 4.76 & 4.76 & 1.41 & 0.35 & 0.21 \\
\hline & 2.0 & 14.5 & 5.56 & 1.54 & 0.07 & 0.04 & 62.1 & 11.1 & 2.94 & 0.07 & 0.03 \\
\hline \multirow[t]{3}{*}{ O4 } & 0.5 & 1.32 & 1.22 & 1.02 & 0.81 & 0.55 & 1.61 & 1.22 & 1.02 & 0.85 & 0.48 \\
\hline & 1.0 & 4.76 & 2.17 & 1.19 & 0.64 & 0.20 & 8.33 & 3.45 & 1.61 & 0.45 & 0.15 \\
\hline & 2.0 & 40.3 & 7.14 & 1.59 & 0.22 & 0.01 & $>100$ & 22.5 & 1.04 & 0.04 & 0.01 \\
\hline \multirow[t]{3}{*}{ O5 } & 0.5 & 1.43 & 1.22 & 1.03 & 0.66 & 0.56 & 1.47 & 1.47 & 1.08 & 0.63 & 0.54 \\
\hline & 1.0 & 5.56 & 3.85 & 1.08 & 0.36 & 0.22 & 5.56 & 2.86 & 1.05 & 0.40 & 0.34 \\
\hline & 2.0 & 42.9 & 4.17 & 1.10 & 0.22 & 0.01 & $>100$ & 7.69 & 1.85 & 0.04 & 0.01 \\
\hline \multirow[t]{3}{*}{$\mathrm{O} 7$} & 0.5 & 1.56 & 1.22 & 1.03 & 1.02 & 0.50 & 1.89 & 1.22 & 1.04 & 1.04 & 0.35 \\
\hline & 1.0 & 5.88 & 1.45 & 0.96 & 0.96 & 0.22 & 3.70 & 1.85 & 0.96 & 0.93 & 0.10 \\
\hline & 2.0 & 54.1 & 2.38 & 2.38 & 1.10 & 0.09 & 54.9 & 4.76 & 1.89 & 0.69 & 0.15 \\
\hline
\end{tabular}

Note-The criterial boundaries for each level of difficulty (ideal-observer $d^{\prime}=0.5,1.0$, and 2.0) have been grouped for each observer, to allow easy comparison.

\section{Decision Space Analyses}

It was the decision space analysis that we hoped would reveal the decision strategy adopted by the observers. Although the strength of evidence from the three experiments is not homogenous, there appears to be a clear outcome. The observers adopted an unsophisticated difference decision strategy when making $\mathrm{ABX}$ decisions about the stimuli that we employed. The three stimulus types included simple spatial (circles) and temporal (tones) stimuli, as well as complex symbols (numbers). More interesting, these observers continued to use this decision strategy for at least 1,000 observations with feedback. Indeed, if we consider that 4 of the 6 observers in Experiment 3 participated in all three experiments, they were still using this suboptimal strategy after 4,000 observations.

The results of the decision space analysis for the first two experiments were clear and unequivocal. The difference decision strategy provided a significantly better account of the data than did the independent-observations strategy. This is a surprising finding because it seems plausible that observers would seek the best possible performance and use the independent-observations strategythat is, unless there is something about the method of stimulus presentation that prevents them from doing so. An example would be the use of a roving experimental design (Macmillan \& Creelman, 1991, p. 133). In the present experiments, there was no impediment to the use of the independent-observationsstrategy imposed on the observers. Yet, they never learned to use that strategy. Rather, they employed a strategy that yields worse performance.

Of the three experiments conducted, Experiment 2 was the most likely to facilitate the use of an independent- observations strategy. This is because it is possible to adopt explicit numerical criteria when the stimuli are numbers and individual stimuli can be directly compared with these criteria. Indeed, previous research with the same-different task (Irwin \& Hautus, 1996) found that some observers were able to adopt the independent-observations strategy for this type of stimulus. Perhaps the observers in that study were more knowledgeable about decision strategies (many were highly experienced observers), or the adoption of an independent-observations strategy is less demanding in the same-different task than it is in the ABX task. The answer is not clear.

The results of Experiment 3 appear a little anomalous, in that statistically significant differences were not found between the performances of the two strategies. However, as was noted, the differences between the values of pcc for each model possessed the same sign for 16 of the 18 comparisons. An interesting point here is that the effect size for the auditory task is much smaller than those for the other two tasks. This suggests, in combination with the overall lower performance of the observers in Experiment 3 , that the auditory task yielded noisier data. Figure 7 illustrates the decision space, based on the difference strategy, for $\mathrm{O} 1$ at the intermediate level of difficulty of the auditory task. A comparison of Figure 7 with Figure 5 (circles) clearly reveals the greater variability in the observer's judgments on the auditory task.

Two obvious distinctions can be made between this experiment and those that preceded it: (1) The stimuli were separated temporally rather than spatially, and (2) the task was time limited - for the first two experiments, the stimuli remained visible until the observer made a re- 


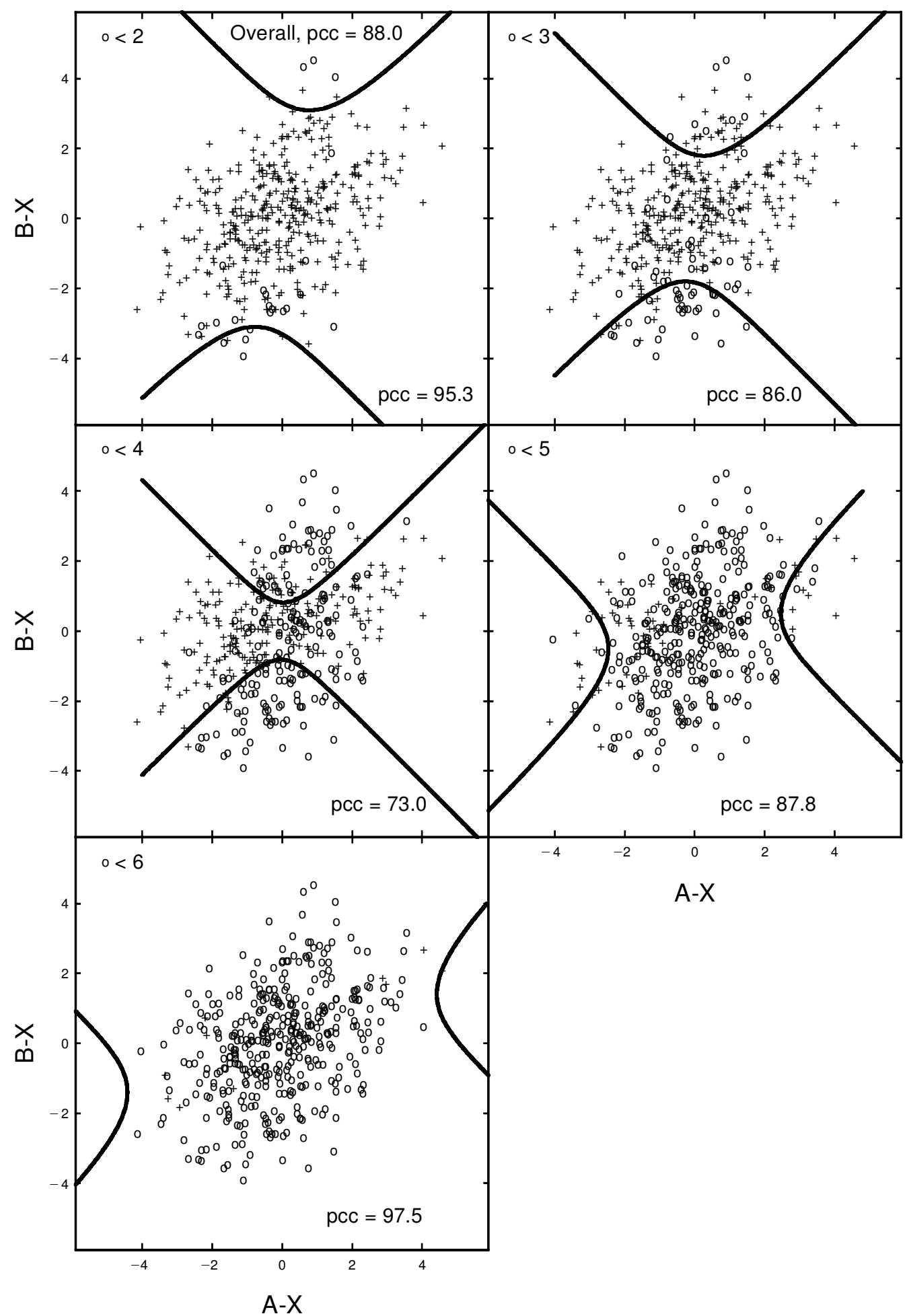

Figure 7. The decision space, based on the difference strategy, for $\mathrm{O1}$ at the intermediate level of difficulty in the amplitude discrimination task of Experiment 3. Each panel illustrates the responses of the observer determined by a different criterial boundary that corresponds to a specific rating response (see the top left of each panel for details). For example, in the top-left panel, open circles correspond to a rating of one (certain the focus was a match to $A$ ), and crosses correspond to all other ratings. The heavy curves are the best-fitting criterial boundaries, and the associated fit statistic is shown in the lower right of each panel. The overall fit statistic is also provided. Comparison of these data with those in Figure 6 reveals the presence of considerably more variation in the responses made by this observer in Experiment 3. There was a similar pattern for all the observers. 
sponse. In combination, these two distinctions would place a greater load on memory processes, leading to the noisier data obtained.

Although it is clear that the decision space analysis method can help uncover the decision strategy adopted in psychophysical tasks, whether the method itself influences the decision strategy adopted is not known. In most psychophysical tasks, the performance-limiting noise is a product of the sensory system itself. However, there are some "natural" tasks in which the limits of performance are defined by the characteristics of the stimulus. One example is the amplitude discrimination of narrow-band noise processes, such as Rayleigh noise (Hautus \& Irwin, 1992). Thus, the introduction of noise by the experimenter may not be so unnatural after all. This issue is open to debate and will be resolved only after further research.

\section{Conclusion}

The results of all three experiments suggest that humans initially rely on the degree of similarity (or dissimilarity) between observations when making decisions. This seems to be a strategy that requires little cognitive effort. Perhaps later, after a great deal of practice, they may discover a better approach - an independent-observations strategy. What is it that makes the independent-observations strategy more "difficult" to use in the ABX task? Perhaps it is a matter of knowledge. To use this strategy, the observer must recognize the independence of the information available from the observations at A and B from the information available at X. Without this insight, at either an implicit or an explicit level, the independent-observations strategy cannot be adopted. The use of the difference strategy requires no distinctions of this kind to be made. Perhaps the difference strategy is the lowest level approach to decision making in the ABX task, and one that most observers do not advance beyond. This suggests that any researchers using the $\mathrm{ABX}$ task, who are not in a position to determine the decision strategy adopted by their observers, could assume the use of the difference decision strategy unless there is convincing evidence to the contrary.

\section{REFERENCES}

Abramowitz, M., \& Stegun, I. A. (1972). Handbook of mathematical functions: With formulas, graphs, and mathematical tables (9th ed.). New York: Dover.

Calder, A. J., Young, A. W., Perrett, D. I., Etcoff, N. L., \& RowLAND, D. (1996). Categorical perception of morphed facial expressions. Visual Cognition, 3, 81-117.

Dupoux, E., Pallier, C., Sebastian, N., \& Mehler, J. (1997). A distressing "deafness" in French? Journal of Memory \& Language, 36, 406-421.

Elliotr, P. B. (1964). Tables of $d^{\prime}$. In J. A. Swets (Ed.), Signal detection and recognition by human observers: Contemporary readings (pp. 651-664). New York: Wiley.

Etcoff, N. L., \& Magee, J. J. (1992). Categorical expression of facial expression. Cognition, 44, 227-240.

Hautus, M. J., \& IRWIN, R. J. (1992). Amplitude discrimination of sinusoids and narrow-band noise with Rayleigh properties. Perception \& Psychophysics, 52, 53-62.
Huorth, J. S. U. (1994). Computer intensive statistical methods. London: Chapman \& Hall.

Irwin, R. J., \& Hautus, M. J. (1996). Exposing the decision space for judgments of identity and difference. In S. C. Masin, Proceedings of the Twelfth Annual Meeting of the International Society for Psychophysics (pp. 287-292). Padua: International Society For Psychophysics.

Kaplan, H. L., Macmillan, N. A., \& Creelman, C. D. (1978). Tables of $d^{\prime}$ for variable-standard discrimination paradigms. Behavior Research Methods \& Instrumentation, 10, 796-813.

Liberman, A. M., Harris, K. S., Hoffman, H. S., \& Griffith, B. C. (1957). The discrimination of speech sounds within and across phoneme boundaries. Journal of Experimental Psychology, 54, 358-368.

Macmillan, N. A., \& Creelman, C. D. (1991). Detection theory: A user's guide. Cambridge: Cambridge University Press.

Macmillan, N. A., Kaplan, H. L., \& Creelman, C. D. (1977). The psychophysics of categorical perception. Psychological Review, 84, 452-471.

NoreEn, D. L. (1981). Optimal decision rules for some common psychophysical paradigms. In S. Grossberg (Ed.), Mathematical psychology and psychophysiology: Proceedings of the Symposium in Applied Mathematics of the American Mathematical Society and the Society for Industrial and Applied Mathematics (Vol. 13, pp. 237280). Providence, RI: American Mathematical Society.

Pastore, R. E., Li, X.-F., \& Layer, J. K. (1990). Categorical perception of nonspeech chirps and bleats. Perception \& Psychophysics, 48, 151-156.

Pierce, J. R., \& Gilbert, E. N. (1958). On AX and ABX limens. Journal of the Acoustical Society of America, 30, 593-595.

Press, W. H., Teukolsky, S. A., Vetterling, W. T., \& Flannery, B. P. (1992). Numerical recipes in C: The art of scientific computing (2nd ed.). Cambridge: Cambridge University Press.

Robinson, D. E., \& Watson, C. S. (1972). Psychophysical methods in modern psychoacoustics. In J. V. Tobias (Ed.), Foundations of modern auditory theory (Vol. 2, pp. 101-131). New York: Academic Press.

Shao, J., \& Tu, D. (1995). The jackknife and bootstrap. New York: Springer-Verlag.

SPENCE, K. W. (1937). Differential responding in animals to stimuli varying within a single dimension. Psychological Review, 44, 430444.

SwETS, J. A. (1986). Indices of discrimination or diagnostic accuracy: Their ROCs and implied models. Psychological Bulletin, 99, 100117.

Tanner, W. P., JR., \& Birdsall, T. G. (1958). Definition of $d^{\prime}$ and $\eta$ as psychophysical measures. Journal of the Acoustical Society of America, 30, 922-928.

\section{NOTES}

1. A variation of the $A B X$ task is the matching-to-sample task. The difference is in the order of stimulus presentation, with matching-tosample being an XAB task. All of the models described in this paper refer equally to the $\mathrm{ABX}$ and the matching-to-sample tasks.

2. The yes/no task is sometimes referred to as a single-interval forced-choice task.

3 . Note that $\beta\left(X_{1}, X_{2}, d^{\prime}\right)$ is the function for $\beta$ with $X_{1}, X_{2}$, and $d^{\prime}$ as parameters. This format is used throughout this article to represent functions.

4. Setting a Type I error rate of .05 could be considered stringent for this type of test. It is plausible that the measurement errors are not normally distributed, having the effect of inflating the value of chi-square. For this reason, Press, Teukolsky, Vetterling, and Flannery (1992) suggested that models for which $p>.001$ should often not be rejected. However, to avoid complication without detracting from our message, we use the value of .05 consistently as a criterion for goodness of fit.

(Manuscript received July 13, 2000; revision accepted for publication January 25,2001 .) 